\title{
The 45th Annual Meeting of the European Society for Blood and Marrow Transplantation: Nurses Group - Poster Session
}

C Springer Nature Limited 2019

\author{
24-27 March 2019 Frankfurt, Germany
}

Modified and published with permission from https://www.ebmt.org/annual-meeting

Sponsorship Statement: Publication of this supplement is sponsored by the European Society for Blood and Marrow Transplantation. All content was reviewed and approved by the EBMT Committee, which held full responsibility for the abstract selections.

Presenting author names are bold type in the contributor lists.

\section{NP001 \\ Decrease Of Hematology Nurse's Exposure To Antineoplastic Drugs}

Gihan Amariya Abu Kamir', Marina Furer ${ }^{1}$

${ }^{1}$ Rambam Health Care Campus, Haifa, Israel

Background: Hematology nurses care for patients who have various types of blood related disorders such as leukaemia, lymphoma, sick-cell anaemia, Hodgkin's disease and haemophilia. Some of the hemtology nurse duties may include obtaining IV access, ensuring that medications are given on time, infusing certain medications like high dose chemotherapy as conditioning before bone marrow transplantation, biological drugs, and another antineoplastic drugs (AD). Antineoplastic drugs, also known as chemotherapy, cytotoxic, and oncology drugs, are used extensively in health care to treat hematological patients who underwent bone marrow transplantaion and are increasingly being used to treat arthritis, multiple sclerosis, and other noncancer medical conditions. Most antineoplastic drugs are hazardous drugs.

In the United States eight million healthcare workers are estimated to be exposed to chemotherapy annually, nurses being among the groups with the highest incidence of exposure. Even a small exposure to antineoplastic drugs can cause adverse outcomes, including skin rashes, nausea, hair loss, abdominal pain, nasal sores, allergic reactions, skin or eye injury, and dizziness. Healthcare workers incur exposure on a repeated basis and often for many years. Chronic effects linked with exposure include reproductive harm, such as delayed time to conception, spontaneous abortion, genotoxic changes.

Safe-handling practices, such as the use of personal protective equipment (PPE) by staff, are known to reduce exposure and likelihood of health effects from chemotherapy (National Institute for Occupational Safety and Health [NIOSH], 2004). Such guidelines recommend workers wear PPE (including double gloves, goggles, and protective gowns) for all activities associated with drug administration and disposal of all equipment used to administer drugs.

Studies have found inconsistent use of ingineering controls, personal protective equipment, and precautionary work practices. The purposes of this study were (i) examine relationships among factors that are believed to affect nurses' use of AD safe-handling precautions; (ii) identify factors that promote or interfere with HD precaution use.

Methods: 30 nurses handling chemotherapy were participated in the study. Instruments: 1. Revised Hazardous Drug Handling Questionnaire (Martin \& Larson, 2003). 2. Chemotherapy Exposure Knowledge scale, the Barriers to Using PPE scale, a self-efficacy scale, and three items about perceived risk. 3.The Workplace Safety Climate questionnaire.

Results: Exposure knowledge, self-efficacy for using personal protective equipment, and perceived risk of harm from AD exposure were high; total precaution use was low. Nurse characteristics did not predict AD precaution use. - 
Fewer barriers, better workplace safety climate, and fewer patients per day were independent predictors of higher $\mathrm{AD}$ precaution use. Better workplace safety climate was associated with higher self-efficacy, fewer barriers, higher perceived risk, lower conflict of interest, and more positive interpersonal influences.

Conclusions: Workplace enviroment interfere with nurses' use of AD precautions. The organizational unit is a significant driver of PPE use during chemotherapy handling. These findings suggest focusing on organizational, rather than individual, factors when encouraging the use of PPE.

Disclosure: Nothing to declare

\section{NP002 \\ Effect of PET-CT prior to peripherally inserted central catheter (PICC) in lymphoma patients:A historical control study}

\author{
Yi Peng ${ }^{1}$, Huiju $X u^{1}$, Liyuan Zheng ${ }^{1}$ \\ ${ }^{1}$ Jilin University, Changchun, China
}

Background: Lymphoma patients have the risk of successful catheterization due to tumor occupying. Traditional vascular ultrasound assessment cannot meet clinical needs. CTV examination is not a routine project. PET-CT is necessary for the prognosis evaluation of lymphoma patients, we tried to improve the preoperative assessment of PICC catheterization and the multi-disciplinary team (MDT) nursing consultation mode, and introduce PET-CT into the preoperative evaluation of PICC catheter implantation without increasing the cost. Therefore, in this study, we tried to evaluate the effect of PET-CT prior to peripherally inserted central catheter (PICC) in lymphoma patients.

Methods: We evaluated success rate of catheter, indwelling time and main complications of PICC in 156 lymphoma patients received PICC according to PET-CT in January 1, 2017 to December 31, 2017. As a historical control,147 lymphoma patients received PICC according to traditional vascular ultrasound examination in January 1, 2016 to December 31, 2016.

Results: In the observation group, the one-time success rate of catheterization, the average indwelling time and the rate of acute phlebitis are $96.79 \%, 85.55 \pm 36.99$ days and $8.16 \%$, respectively. In the control group, the one-time success rate of catheterization, the average indwelling time and the rate of acute phlebitis are $76.19 \%, 45.51$ \pm 12.90 days and $10.20 \%$, respectively. The one-time success rate of catheterization is significantly increase $(\mathrm{P}<$ $0.001)$. Indwelling time is dramatically longer $(\mathrm{P}=0.029)$.
More importantly, the rate of acute phlebitis is decrease $(\mathrm{P}=0.012)$.

Conclusions: PET-CT evaluation prior to PICC increases success rate of catheter, prolong the indwelling time and reduces the incidence of acute phlebitis and vein thrombosis.

Disclosure: Nothing to declare.

\section{NP003}

Abstract already published.

NP004

Probability of finding an HLA-matched related donor after performing extended family HLA typing in highly consanguineous population

Noha Alneedy ${ }^{I}$,Hind Sallam ${ }^{I}$, Rodaina Abujoub ${ }^{I}$

King Abdullah Specialist Children's Hospital, Riyadh, Saudi Arabia

Background: The use of HLA genotypically matched sibling donor is the ideal source of stem cells in allogeneic hematopoietic stem cell transplant. However, the probability of finding HLA matched siblings ranges between $30 \%$ in the western countries to nearly $60 \%$ in Saudi Arabia (Tiercy, Haematologica 2016 and Jawdat et al. BBMT 2009). Performing donor search among extended family members particularly in highly consanguineous population such as Saudi Arabia might increase the chance of finding an HLA matched related donors. In this study, we estimated the probability of identifying HLA matched related donor among extended family members at our institution.

Methods: Our institutional practice for patients who lack HLA matched immediate family members is to conduct donor search in unrelated donor registries and cord blood banks or choose haploidentical donors depending on patient's diagnosis. In addition, we perform extended family typing for patients who do not require urgent donor availability. We retrospectively reviewed the chance of finding an HLA matched related donors among patients who underwent extended family HLA typing between January 2010 and October 2018.

Results: Extended family typing was performed in 52 pediatric patients as shown in the table below. The chance of finding 10/10 or 9/10 HLA matched related donor was $15 \%$. A total of 524 extended family members were typed. Among patients with no history of parental consanguinity, one patient $(7 \%)$ had a matched extended family donor compared with $7(18 \%)$ patients in the presence of parental consanguinity although this did not reach statistical 
significance. Relationship of extended family matched donors were 4 uncles, 3 cousins, and one aunt. Age, number of typed family members, and duration of extended family search did not influence the probability of finding a donor.

Conclusions: The probability of finding an HLA matched extended family members was $15 \%$ among patients with benign disorders who had no HLA matched immediate family members. Presence of parental consanguinity might increase the probability of finding a matched extended family member. There is a need to develop cost-efficient workflow in the clinic and HLA lab for screening extended family members.

\begin{tabular}{ll}
\hline Characteristics & Number of patients = 52 \\
\hline Diagnosis & $\begin{array}{l}\text { Sickle cell anemia (31\%), Aplastic } \\
\text { anemia/Fanconi anemia }(25 \%), \\
\text { Thalassemia (19\%), Other (25\%) }\end{array}$ \\
$\begin{array}{l}\text { Median age in years (range) } \\
\text { Gender (male/female) }\end{array}$ & $33(63 \%) / 19(37 \%)$ \\
$\begin{array}{l}\text { Median number of extended } \\
\text { family tested (range) }\end{array}$ & $7(1-38)$ \\
$\begin{array}{l}\text { Parental consanguinity (yes/no) } \\
\begin{array}{l}\text { Median duration of extended } \\
\text { family search in days (range) }\end{array}\end{array}$ & $38(73 \%) / 14(27 \%)$ \\
$\begin{array}{l}\text { Probability of finding an HLA } \\
\text { matched related donor from } \\
\text { extended family }\end{array}$ & $15 \%(11 \% 10 / 10$ and 4\% 9/10) \\
\hline
\end{tabular}

[[NP004 Table] 1. Table: Probability of finding an HLA matched donor after extended family typing]

Disclosure: None

\section{NP005 \\ Nursing challenges caring for CAR T patients with CRS: A case report}

\section{Paolo Alberti ${ }^{1}$, Jessica Pozzoli ${ }^{1}$ \\ ${ }^{1}$ Fondazione Monza e Brianza per il Bambino e la Sua Mamma, Monza, Italy}

Background: In Monza's AIEOP centre a phase 1/2 trial is underway for the treatment of chemiorefractary acute lymphoblastic B-line leukemia with autologousl $\mathrm{T}$ cells modified ex vivo to express the Chimeric Antigen (CAR-T). CAR-T cells are an immunotherapy used to target the CD19 receptor expressed on the surface of normal and leukaemic $\mathrm{B}$ cells. CAR-T cell therapy is a potent immunomodulatory agent that has the potential to cause serious or lifethreatening toxicity. The most common adverse event after CAR-T infusion is cytokine release syndrome (CRS).
Cytokine release syndrome is a non-specific antigen toxicity that occurs as a result of immune activation. Hematology nurses play a key role in monitoring patients and in assessing the early signs and symptoms of CAR T cell toxicity.

Methods: In the ongoing Phase 1 clinical trial, five patients with CD19 tumor expression detected in bone marrow or peripheral blood were enrolled and treated, between the ages of 3 and 21 at the time of initial diagnosis. Following a bibliographic research in international databases, there was a lack of inherent scientific nursing material.

A nursing tool was developed for the monitoring of Nursing Assistance Needs, according to the method used in the Centre for Care Planning (Marisa Cantarelli Nursing Performance Model) with nursing approach to Primary Nursing; in the AIEOP center of Monza, in fact, every patient has a reference nurse for the personalized assistance approach.The daily use of this tool by the nurse of reference, has allowed to customize and program assistance to the patient undergoing therapy with genetically modified products.

Results: Thanks to the elaborated tool it was possible to draw up a case report of the treated patients emphasizing the nursing assistance needs detected daily. The descriptive study highlighted macro areas of common needs highlighted in all five patients treated; four out of five patients developed CRSs of a higher grade than I, with the need for pharmacological cardiocirculatory support, continuous vital parameters monitoring, respiratory support, auxiliary peripheral access for therapeutic support. In particular, all the assays showed hyperthermia; three out of five patients experienced comitial crises with the need to implement drug therapy; four out of five patients needed oxygen therapy and NIV for tachypnea and desaturation; three out of five patients experienced hypotension with need for pressure support; in all patients it was found important idric retention treated with diuretics.

Conclusions: With the interpretation of the data collected on the five cases examined, we highlighted primary nursing needs common to all patients treated, in particular concerning the cardiocirculatory areas, safe environment, breathing and urinary elimination, showing a greater complexity welfare. With the nursing approach of the Primary Nursing, the caring of these patients required a higher assistance minutage.

The classification of complications related to CRS has allowed the production of an operative instruction for its management and for nursing assistance. The analysis of the assistance complexity from the drafting of the integrated assistance plans will be able to provide useful data for the evaluation of the assistance minutage of the patients treated with gene therapy.

Disclosure: Nothing to declare 


\section{NP006}

\section{The evolution of a paediatric extra-corporeal photopheresis (ECP) service}

\section{Jennifer Lawrence ${ }^{I}$, Julie Guest ${ }^{I}$}

${ }^{1}$ Great North Children's Hospital, Newcastle upon Tyne, United Kingdom

Background: Extra-corporeal Photopheresis (ECP) was initiated within our paediatric Stem Cell Transplantation (SCT) service in late 2012, following a rejected referral for one of our patients to be treated elsewhere in the UK. The service was implemented by a consultant paediatric immunologist with 2 experienced apheresis nurses. Within the first year of establishing the service, 10 patients were treated for a combination of chronic and acute symptoms, with an overall response rate of just $30 \%$.

Since that time, the service has grown exponentially, with a number of lessons being learned, leading to subsequent changes in our practice.

Methods: A number of challenges have been faced and overcome since service provision began. Particular challenges included venous access and care of lines, anticoagulation, assessing response to treatment, educating ward staff around the importance of blood values prior to undergoing ECP, accommodating private patients, available equipment and staffing levels.

The wider multi-disciplinary team has worked exceptionally well together in order to implement a number of changes in practice to improve both patient care and service efficiency.

Results: Since commencing ECP within our centre, we have achieved JACIE accreditation, as well as Centre for Excellence status.

Thanks to earlier initiation of ECP within our patient group, in the last financial year we carried out 278 procedures on 14 patients, with 8 of those achieving complete resolution of GvHD. Additionally, results of a study into thymic recovery following SCT and GvHD carried out within our centre, have allowed us to conclude treatment early on a number of patients with acute GvHD.

As research into ECP and its potential application progresses, our service is likely to continue to expand. Plans for the future of the service include using ECP to treat children and young people with acute solid organ rejection; implementing a clinical assessment tool for use by nursing staff to ensure service is increasingly nurse-led; and developing a 'child-friendly' information package, to aid understanding of the process of ECP.

Conclusions: We find ECP to be a safe, well-tolerated, effective treatment for children and young people with both acute and chronic GvHD.
Gaining experience and knowledge both over time and through various research projects has led to more favourable patient outcomes, with an improvement in the efficiency of the service as a whole.

Implementing this service for complex patients certainly did not come without its challenges, the lessons we have learned throughout the process have enabled us to develop a quality assured service, viewed as a centre for excellence for low body weight patients.

Disclosure: Nothing to declare.

\section{NP007}

Setting up a paediatric extra-corporeal photopheresis service at Birmingham children's hospital, UK: The virtual team and patient outcomes

\author{
Anna Tailby ${ }^{1}$, Julia Nixon ${ }^{1}$, Sarah Lawson ${ }^{1}$ \\ ${ }^{1}$ Birmingham Children's Hospital, Birmingham, United \\ Kingdom
}

Background: Previously, patients with Graft vs Host Disease (GvHD) at Birmingham Children's Hospital travelled a long distance out of area to receive extra-corporeal photopheresis (ECP) therapy. Some patients were too unwell for the journey and as a result the need for an in-house ECP service was identified. An innovative 'virtual team' was formed to rapidly deliver ECP to critically unwell inpatients. Here we describe the start of the ECP unit at Birmingham Children's Hospital, the population of patients we have treated and the impact ECP has had on patient outcomes.

Methods: Setting up a new ECP service required four key stages; equipment and space, staff and training, patient scheduling, documentation and governance. A 'virtual team' was formed, consisting of nurses from across the Trust who already possessed the specialised skills required to work with extra-corporeal circuits. This was an innovative and collaborative approach as they were recruited on an ad-hoc basis in addition to their full-time roles. Training was provided by Therakos ${ }^{\circledR}$ and staff from the Rotherham ECP unit.

We collected data on all the patients who have received ECP at Birmingham Children's Hospital $(n=20$.) We looked at referral letters and treatment records for demographics, underlying diagnosis, reason for ECP, previous/current GvHD or rejection treatment, GvHD stage and grade, number of ECP cycles received and treatment response.

Results: In three years, we have developed a JACIE accredited service with a medical lead, substantive nursing team and a designated unit. The service now provides around 300 paediatric ECP treatments per year, has treated 20patients and has started to accept referrals from out of 
centre. Acute GvHD is the most common reason for ECP therapy $(60 \%, n=12)$, chronic GvHD is the second most common $(40 \%, \mathrm{n}=8)$ and solid-organ rejection is the least common $(5 \%, \mathrm{n}=1)$. Patient outcomes have been excellent with $64 \%$ achieving a complete response, and those currently on treatment are all showing a partial response so far $(n=4)$. ECP also has a strong steroid-sparing quality.

Conclusions: Patients requiring ECP therapy at Birmingham Children's Hospital are no longer obligated to travel out of centre for this life-saving treatment. A rapid and cost-effective way to introduce a new service is to create an innovative 'virtual team' as it utilises existing specialist skills within a centre. Setting up a new service requires four key stages and excellent team work across different setting. Within three years our service has grown into a specialised unit and has achieved JACIE accreditation. ECP is an excellent treatment for children with GvHD with a complete response rate of $64 \%$ in our experience and an important steroid-sparing effect.

Disclosure: Nothing to declare

\section{NP008}

The role of the research nurse in chimeric antigen receptor $T$ (CAR-T) cell therapy

\section{Rose Ellard ${ }^{1}$, Orla Stewart ${ }^{1}$}

${ }^{1}$ King's College Hospital NHS Foundation Trust, London, United Kingdom

Background: CAR-T cells are a new cancer treatment, where T cells (usually from the patient, but sometimes from a donor) are modified in a laboratory to target cancer cells. There have been some promising results in early phase clinical trials and two products are now licensed, one for lymphoma and one for young adults with B-ALL. This could represent a very significant change to the range of treatment options available to certain patients. There are considerable toxicities which need appropriate management. The role of the nurse is crucial in this exciting and innovative field.

Methods: This poster aims to describe the role of a research nurse in a single UK centre.

Results: Clinical: The research nurse acts as keyworker to all trial CAR-T cell patients, taking a key role in coordinating the patient's care and promoting continuity while ensuring patients know who to access for information/ advice in relation to their treatment. Communication with referring hospitals is an important part of the role. Some trials may have waiting lists, meaning patients may be managed in their local hospitals whilst awaiting a slot. As nationwide referrals are received, some pre-treatment investigations may be performed by referring hospitals under the direction of the trial team. Managing expectations can be challenging given coverage of CAR-T cells in mainstream and medical press, giving patients/families high hopes this treatment will offer a cure where other treatments have failed. The research nurse is involved in administering CAR-T cells, including ensuring patients have received appropriate pre-medications. Education: Educating and training nurses is essential to enable effective care and management of this complex treatment. The research nurse has implemented and coordinates a ward nurse teaching programme, including management of cytokine release syndrome and neurological toxicity. Teaching has extended to wider nursing team members, including clinical nurse specialists, advanced nurse practitioners and the intensive care outreach team. As the key worker for this group of patients a major role is the education of patients and their carers in the prompt recognition of toxicities. Research: The research nurse coordinates the CAR-T phase 1 trial, ensuring adherence with regulatory requirements. Data collection/entry and reporting of adverse events to the trial sponsor are important aspects of this role. Cascading training on protocol amendments to all team members involved in patient care is also crucial. Quality management: The research nurse contributes to the production/ modification of departmental SOPs for CAR-T cells, preparation for JACIE inspection, including collating essential documents and being available for interview by the inspection team. External: The role of a research nurse in such a specialist area includes contributions to EBMT cellular therapy working party, ensuring up to date nursing guidelines, sharing learning by submitting abstracts and speaking at national and international study days/ conferences.

Conclusions: This poster explains the role of a research nurse in CAR-T cells in a single UK centre. CAR-T cells are being used in many centres throughout Europe, there is potential for the nursing role to continue to develop in clinical, research, education and leadership roles within the field of CAR-T cells.

Disclosure: Rose Ellard - Molmed (honoraria)

Orla Stewart - Gilead (educational), Kite (educational, advisory board), AbbVie (educational, advisory board), Roche (educational)

\section{NP009}

Ten years of peripheral blood stem cell collection using the terumo optia apheresis platform in a paediatric and adolescent apheresis service for high risk neuroblastoma

\section{Julie Guest ${ }^{1}$, Jennifer Lawrence ${ }^{2}$}


${ }^{1}$ Institute of Health and Society, Newcastle upon Tyne, United Kingdom, ${ }^{2}$ Great North Children's Hospital, Newcastle upon Tyne, United Kingdom

Background: In 2009 the Optia platform was licensed for small body weight peripheral blood stem cell collection.

Mobilisation and collection of peripheral blood stem cells (PBSC) in children with High Risk Neuroblastoma continues to be challenging. However the Optia automated programme has allowed us to develop a service where collection of the protocol requested cell dose of $6 \times 10^{\wedge} 6$ per kilo is now an expectation. This group are primarily small body weight have had a significant treatment pathway following diagnosis, have poor peripheral venous and require high levels of specialist care to enable cell collection.

Methods: A ten year retrospective analysis of the High Risk Neuroblastoma population within our Clinical apheresis service has been undertaken.

Results: Since the service has evolved we have not required autologous bone marrow collection for this patient group.

The development of a specialist low body weight, clinical apheresis nursing service has increased the collection efficiency for this group of children. High risk neuroblastoma treatment is aggressive and this along with patient presentation, bone marrow involvement, and wide spread disease can lead to challenges for mobilisation and collection of cells within the paediatric group.

Optimising collection efficiency is seen as key in the success of our service. Priority is to enable collection of cells on optimal CD34 mobilisation; service provision has been developed to enable some flexibility around mobilisation. Priority of the service has been to minimise the impact of the apheresis procedure on this paediatric population. This has been achieved by minimising collection days, patient exposure to ACDA and reducing collection days and time spent on the apheresis machine. Increasingly patients only require one day of apheresis to collect the cell target.

Conclusions: Over the past ten years there has been a significant increase in the survival rate of children with High Risk Neuroblastoma. Ongoing development of our collection efficiency targets and optimisation of the apheresis procedure enables us to achieve target collection.

Current protocols prioritise collection of cells to enable two autologous transplant procedures. Successful collection following Day 70 chemotherapy is identified within our service as an expected outcome.

Disclosure: Nothing to declare

\section{NP010 \\ Potential benefit and harm of fecal microbiota transplantation for the treatment of intestinal graft versus host disease: An educational intervention}

\author{
Nimer Khatib $^{1}$, Sigal Torati ${ }^{1}$ \\ ${ }^{1}$ Sheba Medical Center, Ramat Gan, Israel
}

Background: Steroid-resistant (SR) intestinal acute graft versus host disease (aGVHD) is a devastating complication of allogeneic hematopoietic stem cell transplantation; effective treatments are lacking. Recent studies have revealed that the microbial composition of the gut (i.e., microbiota) plays a vital role in intestinal aGVHD. Therefore, modulation of the gut bacterial populations using fecal microbiota transplantation (FMT) may be beneficial for treatment of aGVHD. In a pilot study performed at our department, the safety and efficacy of FMT for treatment of SR intestinal aGVHD is being prospectively evaluated. Nine patients have been enrolled to date. To familiarize the nursing staff with the FMT procedure, we developed an educational intervention and evaluated the staffs' knowledge before and after the intervention.

Methods: We constructed a questionnaire evaluating knowledge on the rational, protocol, safety, and potential adverse events of FMT for the treatment of GVHD. Nurses answered the questionnaire before and after educational, which included a lecture by the principal investigator of the FMT study and circulation of a nursing care plan. The change in the staffs' knowledge was quantified.

Results: A total of 20 nurses from the Bone Marrow Transplantation department participated. $75 \%$ knew to define what is the microbiota before the intervention and $100 \%$ after. $65 \%$ thought there might be a relationship between the gut microbiota and intestinal GVHD before compared to $100 \%$ after. $65 \%$ knew the aim of FMT before compared to $100 \%$ after. $45 \%$ were familiar with the methods of FMT administration compared to $100 \%$ after. $25 \%$ knew that infection might be a potential complication of FMT compared to $100 \%$ after. $90 \%$ knew how to report response to FMT before compared to $100 \%$ after. $65 \%$ knew that an antibiotic window is recommended before the procedure compared to $100 \%$ after. $70 \%$ knew that FMT was offered to patients with SR GVHD compared with $100 \%$ after. 55\% knew that broad-spectrum antibiotics could raise the incidence of intestinal GVHD compared with $100 \%$ after.

Conclusions: Our educational program improved nursing staff familiarity with FMT for the treatment of intestinal aGVHD. We believe that such improvement may enhance patients' safety by promoting early detection of complications. A better understanding of the role of FMT 
may improve nurses' cooperation with the investigators and contribute to accurate and reliable documentation and consequent reporting of the procedure outcomes.

Disclosure: No conflict of interest

\section{NP011}

Increasing the impact of meaningful patient research Building a deeper understanding of the lifeworld and burden of illness in a child and adolescent clinical apheresis community

Julie Guest ${ }^{1}$, Roderick Skinner ${ }^{2}$, Eileen Kaner ${ }^{3}$, Andrew Gennery ${ }^{4}$, Karen Heslop ${ }^{3}$

${ }^{1}$ Newcastle, Institute of Health and Society, Newcastle, United Kingdom, ${ }^{2}$ Newcastle, Northern Institute of Cancer Research, Newcastle upon Tyne, United Kingdom, ${ }^{3}$ Newcastle, Institute of Health and Society, Newcastle upon Tyne, United Kingdom, ${ }^{4}$ Newcastle, Institute of Cellular Medicine, Newcastle upon Tyne, United Kingdom

Background: This qualitative study aims to explore the lived experience of children, young people and their families, faced with the further burden of complex novel invasive treatments that have uncertainties and outcomes that may be life threatening. By putting young patients and families at the heart of this project we will enable in-depth exploration through interview based field work and thematic analysis to provide insight into their perspectives around apheresis procedures for cell manipulations such as extracorporeal Photopheresis (ECP) and peripheral blood stem cell (PBSC) collection.

Methods: Engagement with young people and their families has been pivotal for the successful development of the methodological and ethical considerations of this study design. This has been established by involving a young people's advisory group (YPAG), initially around the study concept and then further work was undertaken around semistructured interview questions. However within this dynamic it became apparent that the group had little complex health care experience. Therefore engagement with previously treated patients and their families was seen as integral in the development of patient information sheets, consent forms and interview topic guides that would strengthen and frame the scope of the study and meet the needs of this complex group of patients with life limiting condition requiring apheresis procedures.

Results: Given the lack of scientific papers relating to this specific area of study, developing informed interview topic guides and appropriate methodological choices that enable exploration of the lived experiences of this group was highlighted from both YPAG and expert groups.
Information sheets were improved to reflect this contribution. Developing acceptable language within the information sheets was highlighted by both groups for example "scary words for kids," therefore life limiting and complex were removed. Ethical challenges were presented by patient group teenagers, "when I am ill I won't read the information if there is too much" adapting the information sheets to meet the outline for ethical acceptability and the study population was challenging.

Engagement with previously treated patients and their families has been integral to giving validity to the patient information sheets, consent forms and interview topic guides. Ensuring that these are appropriate and will support recruitment to the study.

Conclusions: The PPI consultation journey for this study, helped to shape the focus of the study and was based on an ethical and acceptability standpoint from both the young peoples advisory and expert patient groups.

Disclosure: No known potential conflicts of interest

\section{NP012}

Pre stem cell transplant patient and family seminar

\section{Maresa Farrell}

${ }^{1}$ Barts NHS Trust, London, United Kingdom

Background: Preparing for stem cell transplantation for some patients can be a frightening and uncertain prospect. Patients in our centre often report that they are not fully prepared and often feel alone and isolated. Hence it was decided in 2016 to address these issues. Patients were asked to reflect on their experience of preparing for and undergoing a transplant and to tell us what we could have done better to prepare them. Four key areas were identified; gaining a better understanding of the transplant process, meeting key people involved in their care, meeting others undergoing a transplant and a forum to ask practical questions.

Methods: After discussion with other members of the multidisciplinary team it was decided that a seminar would be the best forum for addressing these key ideas and concerns. The seminar is interactive allowing patients and family members an opportunity to meet one another in a friendly and informal setting to address any concerns about their impending transplant. At the core is the transplant nurse who sets the momentum and facilitates the seminar encouraging participation and ensuring the concerns of patients and family members. The multi-professional seminar consisting of a dietician, physiotherapist, occupational therapist and the transplant nurse last for two hours and is delivered in a nonclinical environment. Patients are 
invited to bring a maximum of two family members or friends. The seminar is delivered every two weeks thus ensuring that patients are able to attend at any point prior to their transplant. Practical presentations from the team including a DVD on relaxation techniques are reinforced with written information including booklets that patients can read and reflect on.

Results: To date we have delivered 60 seminars attended by 287 patients 400 family and friends. Patient and family evaluations show that that the seminar has been able to alleviate anxiety and fears. Patient and family members appreciate meeting other patients going through a similar process and have the opportunity to better understand the transplant process. Having been able to meet members of the transplant team in this setting, to better understand some of the practicalities of transplant including the need for protective isolation and to have their concerns addressed, patients and family members reported that they felt better prepared for the transplant process.

Conclusions: The opportunity to meet with other patients and family members preparing for transplantation is a core part of the seminar helping to reduce some of the isolation patients previously reported. In this safe and less busy environment patients and their families can ask the questions they need to ask. Patients and families continue to be encouraged to attend the seminar, with most patients attending and appreciating this form of support.

Disclosure: Nothing to declare

\section{NP013 \\ New exemplar training programme: Developing expertise in haemato-oncology nursing}

\section{Alex Abiola ${ }^{1}$, Karen Bradley ${ }^{1}$ \\ ${ }^{1}$ Hammersmith Hospital, London, United Kingdom}

Background: The Haematology department at Hammersmith Hospital, Imperial College Healthcare NHS Trust (ICHNT), offers a wide range of malignant and nonmalignant services that includes a large haematopoietic stem cell transplant (HSCT) programme. In addition to the in-patients wards (54-58 beds) there is a busy day care department that is open $7 / 7$ with extended hours from which innovative treatment pathways operate that include ambulatory care, triage and assessment, day pain service for sickle cell patients, chemotherapy and apheresis services. Within this service framework nurses will have the opportunity to gain experience and develop essential clinical skills in caring for a range of haematology patients with variable acuity levels. This includes developing advanced skills in managing patients undergoing HSCT and the associated treatment-related complications. Skills include administration of stem cells and chemotherapy, responding to acute complications, symptom control and end of life treatment interventions. To function effectively and to provide safe and high quality nursing care, it is essential that nurses have the opportunity to develop this specialist skill set. However, it is also important when looking to future service development that nurses have the opportunity to realise their leadership potential so that they may also positively influence quality improvement.

Methods: There is an ever increasing demand to ensure service provision adapts to technological advancements and contributes to service development. However, this is against the backdrop of being faced with significant challenges that include lack of suitably trained personnel including nurses, which also generates fierce competition to retain experienced nurses and to invest in developing nurses that have the potential to become expert clinicians and leaders.

We have developed and implemented band 5 exemplar training programme to ensure that we have progression for our staff nurses to the next level of becoming a charge nurse with the right skills to be able to deliver a high standard person-centred care across the haematology department.

Four junior nurses were enrolled into the exemplar programme, following a robust selection process which included interview and oral presentation.

Each participant has a competency booklet to complete which includes six reflective practises and a teaching presentation. A quality improvement project was required as well as monthly rotation to other areas within haematology before final sign off by our senior haematology nurse.

Results: New Exemplar training programme equipped trainee nurses to move forward into leadership and management roles. This programme also helped to train our nurses to develop into charge nurse role with the right skills set and enabled them to deliver a high quality personcentred care across the haematology department. The programme has assisted to address shortage of experienced haematology nurses by developing existing workforce, thereby having a significant impact in our recruitment and retention and investing in the service for our future.

Conclusions: New Exemplar training programme proved to be a successful approach to develop junior nursing workforce. The programme allowed junior nurses to have strategic awareness of the organisation which will allow them to undertake more senior leadership roles in the future

Clinical Trial Registry: Not Applicable

Disclosure: No Conflict of Interest 


\section{NP014}

\section{Expanding nurse training to support the delivery of immune effector cells}

\section{Ruth Clout $^{I}$}

${ }^{1}$ The Christie NHS Foundation Trust, Manchester, United Kingdom

Background: The stem cell transplant programme at the Christie is dedicated to adult autologous and allogeneic stem cell transplantation. The clinical facility utilizes two purpose built wards (Palatine Ward PTW and The Christie Private Care TCPC). There are two clinical practice facilitators (CPFS) that cover the transplant programme ensuring the JACIE standards are replicated in both areas. The roles are also to meet the needs of new changes in the standards such as immune effector cells. Further development in the NHS with the likely commissioning CAR-T products, for children and young people for ALL and diffuse large B Cell lymphoma in England. Patients will benefit from groundbreaking cancer treatment, the first in what is expected to be a rapidly expanding class of personalised cancer therapies available on the NHS. (NHS England Sept/ Oct 2018).

The Introduction to Stem Cell Transplantation Study Day was developed in 2014 to offer a standardised curriculum of education and practice within the field of HSCT patient care; providing assurance of adequate nurse training required for JACIE accreditation. Core competencies are specified within the standards, and evidence of training in these competencies must be documented. This may be achieved by evidence of in-service training, attendance at conferences, etc. (The European Blood and Marrow Transplantation Textbook for Nurses 2018)

An additional training day has been introduced to complement and expand on the exiting day; these revisions are to incorporate the JACIE standards 6.01 and support the plans of NHS England and clinical trials.

Methods: The CPFs reviewed training programme to ensure that the all staff meets the training requirements set out for the units. This was measured against JACIE standards 6.01 in preparation for delivery of high risk immune effector cell therapies.

Results: The JACIE standards sections B3.7.3.1/2/3 were covered in existing training. Gaps were identified in B3.7.3.4 cytokine release syndrome, macrophage activation syndrome, critically ill pts and organ failure, neurologic toxicity, tumour lysis syndrome, disseminated intravascular coagulation with these running as ad hoc sessions and no formal records. An advanced transplant day programme was designed, focusing on organ toxicities and other toxicities associated with immune effector cell therapies, such as cytokine release syndrome and neurological toxicities.
Conclusions: Attendance will be required from all staff on the clinical facilities. The days are also offered to critical care, CCU outreach, and the clinical research team. These events will be an established part of the education programme supporting the introduction to transplant day. A further recommendation is the introduction of the Bloodwise $\mathrm{E}$ learning for all staff to complete and CAR T eLearning from the Leukaemia \& lymphoma Society (LLS).

It is essential that training evolves staff in their practice of HSCT; ensuring that staff are continually developed in their clinical roles and professional development. It is important that training and competency programmes are structured and ongoing, with

documented evidence of training topics and dates (EBMT 2018). An annual training review will continued to be reported to the quality management meeting monitoring staff compliance in HSCT training.

Disclosure: Nothing to declare

\section{NP015}

Muslim families undergoing a bone marrow transplant in the UK: Are their religious needs being met?

\section{Najma Sharif ${ }^{1}$, Rima Begum ${ }^{I}$}

${ }^{1}$ Royal Manchester Children's Hospital, Manchester, United Kingdom

Background: Religion is perceived as the most significant characteristic of illness; however, it is a subject that is still neglected. Healthcare professionals may not recognise the influence that religion and religious practise has on the experience of Muslim families in hospital. Prayer is a religious practice that is used most whilst patients are undergoing Bone Marrow Transplants (BMT). It was found that knowledge about Muslim prayer, such as the direction to face and resources like a multi-faith room and chaplaincy service allowed families to feel more supported, thus improving the overall BMT journey. To provide the best possible care to Muslim patients and families, it is imperative for healthcare professionals to gain an understanding of their religion, Islam.

Methods: To find out how much staff knew about Islam; a questionnaire was created and given to members of our multi-disciplinary BMT team. Literature reviews were conducted to gain an understanding from previous studies, focusing on the needs of Muslim patients and their experiences as an inpatient. It was also important to learn directly from Muslim patients and their families, if we were meeting their needs and if they were happy with the care they were receiving. Therefore, a verbal interview was conducted with a number of patients and their parents. This 
allowed us to appreciate the experience from their perspective and come to a conclusion about what changes are required to meet their religious needs.

Results: From reviewing literature, it was found that knowledge about Islamic beliefs and experiences enabled healthcare providers to enhance the BMT experience and overall satisfaction of Muslim families.

On analysing the discussion from the interviews which took place with patients and their families, it was highlighted that their religious needs were being met, at least in part. It was evident that families expressed there is room for improvement, for example providing protected prayer times.

The need to increase knowledge about Islam amongst health care professionals was recognised, to improve family centred care.

These findings were further supported by the questionnaires. The results showed that staff also felt that it was important to gain basic understanding of different religions, which they may encounter at work.

Conclusions: Prayer is a fundamental practice which has an impact on decision making, understanding and responding to different situations that may arise during a transplant. At current, protected prayer times, in which families are not disturbed whilst praying are not provided. Therefore, protected prayer times need to be offered. It is evident that educating staff about the significance of prayer is important. Working together with the education facilitator to plan study sessions for staff, to help raise awareness of Islam will improve their understanding.

By addressing these issues and implementing strategies to achieve the required outcomes highlighted from the research, it will hopefully enhance Muslim families experience whilst undergoing a BMT.

Disclosure: Najma Sharif and Rima Begum: Nothing to disclose

\section{NP016 \\ Developing an information package about extra- corporeal photopheresis (ECP) for children}

\author{
Jennifer Lawrence ${ }^{I}$, Julie Guest ${ }^{1}$ \\ ${ }^{1}$ Great North Children's Hospital, Newcastle upon Tyne, \\ United Kingdom
}

Background: Extra-corporeal Photopheresis (ECP) was initiated within our paediatric Stem Cell Transplantation (SCT) service in late 2012. Information for children regarding the process of ECP, as well as advice on aftercare is limited.
The aim of this project was to develop a 'child-friendly' information package for children requiring ECP treatment.

Methods: Our team uses a number of information booklets for children regarding other aspects of their treatment in order to inform and educate them about their treatment journey.

The booklet provided by Therakos was used to assist the development of our information booklet. We knew, however, that in order to ensure children understand the context; we would need to firstly adapt its language, as well as making it a more visually appealing document.

Patients were asked a number of questions about their treatment including what they felt ECP was, and what they felt other children coming for ECP would need to know. Our patients were also asked to draw pictures that could be included in the booklet.

Results: An information booklet for children has been successfully developed, using the information provided to us by patients undergoing treatment.

This has been evaluated by both more recent patients requiring treatment and children not undergoing ECP procedures in order to increase our sample size and ensure the information would be easy to understand for a child who had not yet experienced the process.

Results of the evaluation have been used to update the booklet.

Conclusions: It is equally important to clarify what ECP involves for the child as it is for the consenting adult.

By supporting children with age appropriate treatment specific information we have found that children are more actively involved in their treatment journeys.

We find providing information specifically for children to be hugely beneficial in ensuring patient engagement with their treatment journey.

Disclosure: Nothing to declare.

\section{NP017}

Importance of patient education before mobilisation and stem-cell harvest to prevent collection failure: An apheresis nurse perspective

\section{Sinju Thomas ${ }^{I}$, Rosalinda Elio ${ }^{I}$, Tsembayena Dlamini, Karen Bradley ${ }^{1}$, Frances Sarkari ${ }^{I}$}

\section{${ }^{1}$ Hammersmith Hospital, London, United Kingdom}

Background: Hematopoietic stem cell transplantation (HSCT) is an integral component and often lifesaving treatment for many haematological malignancies and autoimmune diseases. Although there is risk associated with this treatment modality the benefits of HSCT significantly increase patient survival and long-term quality of life. An 
optimal HSC yield is fundamental to the safety and efficacy of HSCT. Minimum threshold for the engraftment of hematopoietic cells is $>2 \times 10^{\wedge}(6) \mathrm{CD} 34+$ cells $/ \mathrm{kg}$. Below this level it is defined as stem cell mobilization failure.

Methods: From March 2017 to March 2018 the HSCT (adult) service at Hammersmith Hospital, Imperial College Healthcare NHS Trust (ICHNT), performed 110 autologous HSCT and 58 allogeneic HSCT using mobilised peripheral blood stem cells. At ICHNT, we use a range of mobilisation protocols that include cyclophosphamide and granulocyte colony stimulating factor (G-CSF), etoposide and G-CSF, G-CSF alone or \pm Plerixafor. All procedures were undertaken using a Spectra Optia Apheresis System in accordance with a standard operating procedure.

At ICHNT, from January 2018 to October 2018, we performed 181 hematopoietic stem cell (HSC) collections and achieved a high proportion of successful target HSC collections. The target cell dose is $>2 \times 10^{\wedge} 6 / \mathrm{kg} 34+$ cells $/ \mathrm{kg}$ patient weight for single autologous procedures (e.g. multiple sclerosis and lymphoma) and $>4 \times 10^{\wedge} 6 / \mathrm{kg} 34+$ cells/ $\mathrm{kg}$ patient weight for more than one autologous procedure (e.g. multiple myeloma). The target cell dose for allogeneic collections was $>5 \times 10^{\wedge} 6 / \mathrm{kg} 34+$ cells $/ \mathrm{kg}$ patient weight. Data was collected retrospectively from electronic patient records and stem cell laboratory records.

Results: There are many risk factors associated with mobilisation failure which includes age, prior exposure to alkylating agents, extensive radiation, bone marrow infiltration and previous mobilisation failure.

The mobilisation protocol can be daunting for some patients/donors due to poor understanding and fear of the procedure. Education plays an important role in supporting patients/donors through this process. Successful treatment outcomes rely on patients/donors being compliant with all elements of the protocol. A lack of understanding may affect compliance in relation to the timings of treatment and correct dosage administered, which can contribute to poor mobilisation results. Poor compliance also has the potential to negatively impact the use of available resources. Ensuring patients are adequately supported during this process has the potential to improve treatment outcomes and overall patient experience.

In the Haematology Apheresis Unit (HAU) at ICHNT all patients scheduled for HSC harvest are seen in advance by a senior apheresis nurse practitioner prior to mobilisation and collection. Patient education needs to be comprehensive and easily understood. Education includes: Orientation to the Apheresis unit/Appointment time for harvest; Explanation about Mobilisation Regime; GCSF teaching to include purpose, side-effects, administration technique/competency, safe storage treatment timings; Venous access assessment; Information on early detection of potential complications and actions to take and Contact details for 24/7 haematology triage assessment unit and the Apheresis unit.
Conclusions: Our HSC collection programme has been successfully implemented due to our multi-disciplinary approach, which incorporates comprehensive patient education and preparation, access to clinical nurse specialists, apheresis nurse practitioners, advanced nurse practitioners, stem cell laboratory staff and transplant clinicians

Clinical Trial Registry: Not Applicable

Disclosure: No conflict of interest .

\section{NP018 \\ Croatian experience with the multdisciplinary team for cgvhd and long term complications after alloHSCT: A nurse`s perspective}

\section{Lucija Jurišicic ${ }^{1}$, Antonela Samardžićc ${ }^{1}$, Ivan Sabol'², Vesna Družinic ${ }^{1}$ \\ ${ }^{1}$ University Hospital Center Zagreb, Zagreb, Croatia, ${ }^{2}$ Institut Ruđer Bošković, Zagreb, Croatia}

Background: Chronic Graft-versus-Host disease (cGVHD) is the leading cause of morbidity and nonrelapse mortality among allogenic hematopoietic stem cell transplantation (alloHSCT) recipients. It is a complex multiorgan disorder with significant adverse effect on patients' quality of life. Multidisciplinary team for cGVHD and long term complications after alloHSCT was established in 2013 at the Division of Hematology, University Hospital Center Zagreb, Croatia, what was the first such team in Europe, in collaboration with the National Cancer Institute, National Institutes of Health (NIH), USA. The primary purpose was to improve diagnostic and treatment options for our patients, but also to contribute in research of complex pathophysiology of the cGVHD.

Methods: Using multidisciplinary approach for last five years, 130 patients (69 males and 61 females) who underwent alloHSCT were assesed and have been monitored regularly since establishment of Multidisciplinary team. Among studied population, the median time from transplant to enrollment was 15.8 months, range [2-318], and median age at entry was 43.5 years [9-71]. Chronic GVHD was confirmed in 80 patients (in 3 cases after second transplantation), and median time from cGVHD diagnosis to enrollment was 59.5 days. During the follow-up period 14 (11\%) patients died. Standard cGVHD scoring included clinical evaluations, extensive laboratory analyses, and filling QOL questionnaires and forms according to NIH Consensus recommendations. Patients were evaluated by hematologist, dermatologist, ophthalmologist, dentist, PT specialist, gynecologist (female only), nutritionist, neurologist, with additional consultation of pulomologist, endocrinologist, gastroenterologist, ID specialist, immunologist, and others if needed. 
Results: In such dynamic multidisciplinary team the role of a well trained nurse is crucial. As the first health professional patient encounters, nurses must be familiar with the course of cGVHD, but also with multidimensional problems patients after alloHSCT can experience. For regularity of check-ups, it is important to recognize psychological, social and financial difficulties related to this disease, and advise patients based on previous experiences. Availability and empathy is also a major requirement for a nurse working in such environment - patient and his family must feel secure and well-informed to proceed with regular check-ups. It is also important to be able to recognise early symptoms of cGVHD and alarm referring specialist. Since working in multidisciplinary team, managment skills are highly demanding - nurse must coordinate a large team involving 20 specialists from different branches of medicine, laboratory tests and other analyses, and make appointments for necessary tests.

Conclusions: Multidisciplinary team for cGVHD and long term complications after alloHSCT in Croatia improved quality of medical management of these longterms survivors with complex and long-lasting health issues, with potential positive impact to health-related costs and benefit to society. The role of nurse in such team is of great importance, not only for managment and coordination of the team, but also for patient compliance.

Disclosure: None

\section{NP019}

\section{Nursing care in mucositis}

Cristina Martín Benito ${ }^{1}$, Silvia Sangüesa Domínguez, ${ }^{1}$, María Cristina González Rodríguez, Francisco José Rodríguez Alcazar ${ }^{2}$, María Rosa Cortés Pinilla ${ }^{1}$, Irene Gaite Sánchez ${ }^{I}$

${ }^{1}$ Complejo Asistencial Universitario de Salamanca, Salamanca, Spain, ${ }^{2}$ Gerencia de Atención Primaria de Salamanca, Salamanca, Spain

Background: Mucositis is an inflammatory reaction that affects the gastrointestinal tract, being one of the main adverse effects due to the toxicity of the antineoplastic treatments (radiotherapy, chemotherapy and bone marrow transplant) used in onco-hematological diseases. To prevent the appearance of this pathology, it is essential that hematological patients perform adequate oral hygiene. We must explain the importance of well- washing all the teeth with a soft bristle brush on the front, back and top. In addition to dental hygiene, it is essential to clean the tongue, gums and mucous membranes gently and gently.
Methods: An exhaustive bibliographic review is carried out through databases such as Medline, Pubmed and Cochrane. The purpose is to unify criteria in the use of rinses.

Results: The use of mouthwashes has beneficial effects in the mucositis' prevention and treatment.

To clean the mouth that has many debris, the patient will be given a solution composed of bicarbonate, bottled water (or distilled) and oxygenated water in equal proportions, to which we can add Chlorhexidine. The bicarbonate is an alkalizing molecule which, in combination with saliva, prevents the overgrowth of aciduric bacteria such as Candida albicans, fluidizes saliva, stimulates the salivary glands and prevents decalcification of the enamel. The oxygenated water, diluted in equal parts in bottled or distilled water, drags the food debris and detritus and crusts that accumulate in the teeth and mucosa. Repeated use of rinses with hydrogen peroxide as prophylaxis or continuously due to potential damage to fibroblasts and keratinocytes is not recommended, affecting the healing process if there is any injury. Therefore, only oxygenated water should be used one or two days, as it could delay the healing of the lesions.

While the patient has full mucosa, Chlorhexidine will be used after meals. If discomfort, pain, speech problems or ulcers appear, Chlorhexidine will be removed and Aloclair ${ }^{\circledR}$ mouth rinses will be applied after meals and an hour after rinsing with nystatin (which will be swallowed if tolerated). Before going to bed, Aloclair ${ }^{\circledR}$ will be applied again because it creates a protective plate in the mouth that will work throughout the night, helping to regenerate the mucosa. If there is perianal irritation, local Aloclair ${ }^{\circledR}$ can be applied on demand.

Conclusions: The role of nursing is fundamental in the care of the mouth and the correct use of mouthwashes, reducing the onset of mucositis, the degree and its complications. A reduction on the incidence of mucositis has been observed in patients who maintained their oral hygiene and performed the rinsings correctly.

It is essential to teach hematological patients to perform proper oral hygiene, maintaining the integrity of the mucosa and making sure to eat, drink and talk without pain, avoiding the risk of infections and the loss of their quality of life. Achieving their well-being, comfort and decreasing sanitary costs.

Disclosure: Nothing to declare

\section{NP020}

Nutritional status and influencing factors in patients after allogenetic hematopoietic stem cell transplantation

Si Cheng ${ }^{I}$, Yun Fang ${ }^{I}$, Minjie Liu ${ }^{I}$, Xianjie Xiao ${ }^{I}$ 
${ }^{1}$ Huazhong University of Science and Technology, Wuhan, China

Background: Nutritional status is privotal in patients after hematopoietic stem cell transplantation, being associated with infection rates.,morbidity and mortality issues. The research on nutriional status of patients underwent alloHSCT is always concentrated in peritranspantation stage but less in post transplantation stage. The objective of this study was to investigate the nutritional status in adult patients after allo-HSCT, to evaluate the incidence rate of malnutrition and to explore its influencing factors.

Methods: A total of 148 patients were recruited from a class-three hospital in Wuhan. They were investigated with a basic information questionnaire, the Patient-Generated Subjective Global Assessment (PG-SGA), triceps skinfold (TSF) and mid-arm circumference measurement, serum albumin, hemaglobin, C-reactive protien and the count of white blood cells, lymphocytes, neutrophils recording. The clinical hematological and nutritional data were collected.

Results: The average score of PG-SGA was (10.54 \pm 4.12 ) for 148 patients, $62.16 \%$ patients suffered from malnutrition and $66.90 \%$ patients suffered from dietary problems. The score of PG-SGA was significantly different with different age, duration after allo-HSCT, classification of primary disease, occcurrence of gastrointestinal GVHD, C-reactive protein, hemoglobin, neutrophil counts and dietary intake $(P<0.05)$. Significant differences were seen in the proportion of different age group and post-HSCT duration group, the number of digestive tract symptom $\geqq 2$, body mass index $(\mathrm{BMI})<18.5 \mathrm{~kg} / \mathrm{m}^{2}$, low serum albumin (ALB) and Hemoglobin(Hb)level, and the gastrointestinal GVHD between the malnutrional group and normal nutrional group $(P<0.05)$. Which were the independent risk factors of malnutrition occurrence by Logistic regression analysis $(P<0.05)$.

Conclusions: There was high incidence rate of malnutriton in adult patients after allo-HSCT and the influencing factors of malnutrition are varied. Clinical professionals should pay attention to the assessment of nutritional status, and give reasonable and strengthened nutritional interventions in time, to improve condition of malnutrition, and promote health.

Disclosure: Nothing to declare.

\section{NP021}

\section{The haematology advanced nurse practitioner: 'A multifaceted role in stem cell transplantation'}

\section{Marie Waller $^{1}$}

${ }^{1}$ Manchester Royal Infirmary, Manchester, United Kingdom
Background: The stem cell transplant unit is busy and challenging with complex patients who have multiple nursing and medical needs.

Historically the roles of nurses and doctors has been well defined. While nurses deliver direct patient care including administration of medications, chemotherapy and blood products the medical team undertake clinical assessment, examination and formulate a medical plan.

Roles such as clinical nurse specialist and transplant nurse coordinator are embedded within the transplant team. These roles often include skills such as non medical prescribing, bone marrow biopsies and placement of PICC lines. The role of haematology advanced nurse practitioner (ANP) is relatively new within this setting.

Within the United Kingdom (UK) the ANP role evolved due to reduction in junior doctors working hours, roles were initially in primary and urgent care. In recent years the role has become established in other clinical areas giving senior nurses an opportunity to further develop their clinical skills, attributes and careers.

Methods: After undertaking the MSc in Advanced clinical practice and non medical prescribing course the author became a qualified ANP working on the stem cell transplant unit.

The ANP works within the medical team and manages a case load of patients; both chemotherapy and stem cell transplant. The role includes clinical examination, assessment and management of side effects following treatment, formulating a medical plan, non medical prescribing and ordering and prescribing of blood products.

The ANP coordinates the junior medical team, facilitates timely patient discharge, ensures smooth running of the transplant unit and provides leadership to nursing and medical colleagues.

The ANP is involved in education and leading change of practice (I.e, developing guidelines) both locally and nationally.

Results: The ANP has become an integral role to ensure the smooth running of the transplant unit. The literature describes the ANP as a linchpin- bridging the gap between the medical and nursing team. This includes communication within the MDT, a point of contact for patients and relatives, educator and role model for nurses and leadership for junior team members.

Within the UK there are four main elements that underpin the ANP role: Advanced clinical practice, Research, Education, and Leadership. The ANP can manage their own clinical workload and undertake thorough holistic assessments using advanced communication and clinical skills. As the ANP is permanent this can improve continuity of care for patients unlike rotational training or middle grade doctors. 
The ANP has become a point of contact for the extended members of the multidisciplinary team such as microbiology, palliative care and pharmacy.

Conclusions: The role of the haematology advanced nurse practitioner has become embedded and integral within the stem cell transplant unit. The multifaceted role incorporates the four elements of advance practice whilst ensuring safe, effective and quality care is delivered to patients on the transplant unit. The ANP can bridge the gap between the medical and nursing team offering support, education and leadership to both. Furthermore the ANP can use advanced clinical skills to holistically assess and manage the complex needs of transplant patients.

Disclosure: nothing to declare

\section{NP022}

\section{The reality - identifying cost improvements in donor} requests and deferrals

\author{
Angela Leather', Rita Angelica ${ }^{1}$, Lindsey Ashton ${ }^{1}$ \\ ${ }^{1}$ Christie Hospital, Manhester, United Kingdom
}

Background: With increasing demands for cost improvement and service development, this first time audit was implemented to identify if improvements could be made in relation to ordering donors, seeking commonalities in the cancellation or deferral of volunteer unrelated donors. It is accepted that some donor changes may be required. Our centre performs approximately 60 - 70 volunteer donor transplants per year.

Methods: Each deferral or cancellation was recorded with reason and timing for deferral, it identified how far in advance the donors were requested. Obtaining accurate finance costings was difficult as it was recorded on invoices which were not accessible and do not get filed under patient/ donor identification, therefore estimated costings were used for deferral, cancellation and repeat blood testing, according to each patient/donor case, this 'standard' cost information was provided by the Anthony Nolan Donor Registry

Results: 27 patients had donor deferrals with 36 total deferrals being sent, 5 patients and 6 donors were deferred on more than one occasion. Of the 27 patients - 11 proceeded to allogeneic transplant and 2 patients had autologous transplantation. Cryopreservation was required in 2 deferrrals, due to time and coordination of alternative dates, however both patients were transplanted with these cells. No donors donated without the product being infused. 1 donor had 4 deferrals, and ended that this donor was then not able/willing to proceed due to social circumstances and a further donor was identifed for the patient. Donors were deferred or cancelled for various reasons - 12 were due to patient infection and 11 disease progression which is a common complication in transplant work up. 3 donors were requested too soon, where patient was not considered to be recovered from induction therapy, 3 donors were cancelled due being inappropriately ordering or where patient circumstances changed, 4 of the cancellations were due to donor sircumstances, having failed medicals or not able to meet requested dates and alternatove donors sought. In 3 instances patients opted to defer or cancel donation either for personal reasons or alternative treatment.

Many of the deferrals came after a donor medical had been performed, increasing cost significantly and inconvenience to the donor. 21 donors had medical reviews and 4 required these required repeat virology bloods, 8 of the recipients were transplanted. 15 donors were cancelled / deferred prior to medical examination.

Conclusions: In times of ongoing financial pressures, it is important to recognise costs improvements and when they can be avoided, whilst route to transplantation is not always straight forward and often changes are needed due to patient complications, this audit highlights the impact when inappropriate requests are made, this audit estimated cost of an additional $£ 62000$ in one year, these costs were purely brought about through deferral and perhaps could have been avoided. This does not take into account hidden costs such as time and efficiency for staff, both in house and at collection centre and registries.

Disclosure: Nothing to Declare

\section{NP023}

Abstract already published.

\section{NP024}

How to establish a bone marrow transplant unit in the absence of a facility for apheresis: A two stop shop

Nurit Avraham ${ }^{1}$, Jacob Rowe', Ayala Lifscytz', Miri Mintz $^{1}$, Tamar Hatuka ${ }^{1}$, Rebeca Jackson ${ }^{1}$, Reut Nebenzal $^{2}$, Zila Zuckerman ${ }^{3}$, Rachel Barchad ${ }^{3}$, Shira Setter ${ }^{3}$

${ }^{1}$ Shaare Zedek Medical Center, Jerusalem, Israel, ${ }^{2}$ Sha'are Zedek Medical Center, Jerusalem, Israel, ${ }^{3}$ Rambam Health Care Campus, Haifa, Israel

Background: Autologous transplants are common and represent a major therapeutic modality, used primarily for treatment of lymphoma, myeloma, acute leukemia and some solid tumors.

Facility for such transplants requires unique physician skills, dedicated nursing staff and transplant coordinators, in 
addition to specialized in-patient facilities with complete tertiary care support services.

Methods: This report describes a partnership among two medical facilities where one facility enables the apheresis to be performed. All else is performed at the other medical center, a dedicated transplant unit.

In September 2013, the Shaare Zedek Medical Center in Jerusalem, Israel, initiated an autologous HSCT program. As on November 2018, 97 consecutive transplant were performed.

The patients follow a special path where the preliminary investigation and diagnosis are done at the Saare Zedek Medical Center. Large gauge Central venous catheters are always inserted by the transplant center. For the apheresis procedure patients are transported to a distant facility that is well equipped and experienced in handling the intricate procedure. Depending on the number of CD34 cells collected, the procedure may be completed in one, two, or, uncommonly, three successive days.

Following hematopoietic cell harvesting, cells are processed and cryopreserved . 24 hours prior to transplant, cells are transported in -180 degrees centigrade to the transplant center. Thawing at 37 degrees is done at the bedside as per standard procedure.

Results: The idea is to go forward with HSCT procedure even if the process is shared between two facilities. Many hospitals may avoid HSCT procedures if they don't have all the equipment to perform both the apheresis procedure as well as the BMT itself.

This experience has proven a success. Engraftment kinetics were excellent and overall clinical results satisfactory despite the inconvenience of travelling to a distant facility for the apheresis procedure. All of this was enabled as a result of unique collaboration between the two medical centers.

No infections related to transportation of cryopreserved cells occurred. Occasional need for Plerixafor (mozobil) presents a challenge, which was always overcome.

Conclusions: Transplants can begin in a suitably staffed medical center despite the absence of an apheresis facility on site.

The distant facility that performs the apheresis and collection of stem cells has an operating room facility if needed.

When both facilities collaborate for the benefit of the patients, the financial burden is shared as well and has not presented an issue.

Our facility has gained the trust and satifaction of the patients. Despite the fact that they may have other options that offer both procedures in one facility- a one stop shop- . Our patients choose our facility and the caring staff that escorts the entire process overlooking all the details, utilizing a map for each patient with clear directions to two stop shop.

Very close collaboration and trust between centers may supersede the hindrance of long distance travel.

Disclosure: No conflict of interests

\section{NP025}

Cerebral toxoplasmosis after haploidentical stem cell transplantation in a patient having many wild cats - a nursing challenge

\section{Yu-Chieh Chen ${ }^{I}$,Jia-Hau Liu}

${ }^{1}$ Tai-Cheng Stem Cell Therapy Center, National Taiwan University, Taipei City, Taiwan, Republic of China

Background: Cerebral Toxoplasmosis is a rare complication but may cause mortality after haplo-identical stem cell transplantation (Haplo-SCT). Here we reported a rare case of Cerebral Toxoplasmosis shortly after Haplo-SCT.

Methods: The patient was a 59-year-old man with diffuse large B cell lymphoma, Ann arbor stage IIIB. He underwent a 8/10 HLA-matched peripheral blood stem cell transplantation from his daughter following posttransplantation cyclophosphamide-based reduced intensity conditioning. He was engrafted on day 22 after the transplantation. Post transplantation course was complicated with a grade II acute graft-versus-host disease of gut, G2S1L0, for which he was treated with one-week of corticosteroid. However, he started having progressive lower leg weakness, ataxia, consciousness drowsy, and intermittent low-grade fever since day 47 . On day 56, a whole body computed tomographic (CT) scan showed no systemic lymphoma relapse but accidentally found multiple enhancing cerebral nodules. Radiologist diagnosed cerebral toxoplasmosis from brain magnetic resonance image (MRI). Serology and PCR test of serum and cerebrospinal fluid (CSF) were all negative for toxoplasma. Trimethoprim/ Sulfamethoxazole was initiated immediately and later substituted with pyrimethamine. An open brain biopsy of the masses showed a positive tissue real time PCR for toxoplasmosis from Center for Disease Control examination. Under toxoplasmosis treatment, the patient had a gradually neurological improvement in the following 2 months and a follow up brain MRI showed $95 \%$ of regression of the brain masses. He remains uneventful for the following 2 years.

Results: At the time when the toxoplasmosis was diagnosed, the families reported many wild cats fostered by the patient in the mountain before the Haplo-HSCT. He also liked to eat medium-well cooked beef. During the diagnosis and treatment of the cerebral toxoplasmosis, the nursing works includes: 1. Contact the CDC and the Infection Control Center for PCR diagnosis and special medication of toxoplasmosis. 2. Holding of family meeting: to educate the segregation with his cats and the necessity to prepare fullcooked food at home. Also we educate the families cleaning after contact with the cats and approaching the patient. 3 . Bring the fostered cats to the veterinary hospital, and take blood test for toxoplasma IgM. 4. Knowing the 
pancytopenia side effect of Pyrimethamine and other Toxoplasmosis medications and alert the physician with regular hemogram follow up. 5. Collaborate with the rehabilitation team to help the patient recover his muscle strength after the treatment. 6. Psychological and religionary support for the family to calm down their anxiety and re-building of their confidence on the transplant team.

Conclusions: Cerebral toxoplasmosis mostly occurs in patients with low immunity. The cat is the only known definitive host of Toxoplasma gondii. The disease is transmitted to humans through the kitten's infected feces. The patient's family raise ten kittens in the house, and he often touch the cat's litter or feces without wearing any gloves, when cleaning the cats' litter. In conclusion, cerebral toxoplasmosis is very rare complications after allo-HSCT. The nurses play an critical role for either care and education for the patient and his family.

Clinical Trial Registry: None

Disclosure: No disclosure of potential conflict of interest

\section{NP026}

Preparing the apheresis service for the introduction and delivery of cellular therapy: Challenges and solutions

\section{Janet Baker', Dee Moloney ${ }^{I}$}

${ }^{I}$ Royal Marsden NHS Trust, Sutton, United Kingdom

Background: Apheresis is the starting point for adoptive cell transfer therapies. The most promising of these are Chimeric Antigen Receptor T cell therapy (CAR-T) for the treatment of haematological malignancies, and will necessitate unmobilised leukapheresis procedures. In the UK, 2 CAR-T products are currently licenced and approved for this indication. In addition, numerous clinical trials are open/ opening, with other tumour groups exploring the use of collecting large numbers of mononuclear / $\mathrm{T}$ lymphocytes for both diagnostic and therapeutic purposes via Apheresis.

Methods: The Apheresis unit primarily serves the Haematopoietic Stem Cell Transplant (HSCT) service at our tertiary referral JACIE accredited cancer centre. Data retrospectively reviewed over an 18 month period (January 2017- June 2018) revealed a total of 377 Apheresis procedures. $82 \%$ were performed for adult autologous HSCT, $3 \%$ for paediatric autologous HSCT, 7\% related allogeneic HSCT, 2\% donor lymphocyte collections, and 2\% Therapeutic Plasma Exchanges. An additional $4 \%$ were diagnostic solid tumour leukapheresis procedures. The question emerged regarding the inclusion of further procedures for cellular therapies into our existing practice. Challenges included space, capacity, scheduling, staffing, and education and competency on aspects of clinical trials.
Results: It remains uncertain how much additional pressure this will create for the current Apheresis service; many patients scheduled for CAR-T therapy would otherwise progress down the autologous HSCT pathway and undergo peripheral blood stem cell collection. However, we required both the flexibility and the capacity to offer patients Apheresis slots without comprising access to trials and precise cellular therapy manufacturing timelines. In response, and to ensure the cancer centre as a whole was capable of delivering CAR$\mathrm{T}$, a cellular therapy steering group was established with Apheresis personnel a key component. Additional space was allocated in the research unit to accommodate these procedures. The most significant outcome has been the introduction of a new nursing role which combines Apheresis with Research, and cell collection with cell re-infusion.

Conclusions: This indication for Apheresis will continue to expand in the future. We therefore have to adapt our current service in order to contribute and participate in this exciting novel therapeutic intervention. This requires exploring different ways of working and innovative approaches to the delivery of care to patients requiring leukapheresis. Our solutions include multi-disciplinary collaboration, use of satellite areas, and the introduction of a new nursing role: the Apheresis/Cellular Therapy Specialist Nurse. This will provide continuity of care for this patient population and enhance patient experience.

Clinical Trial Registry: $\mathrm{n} / \mathrm{a}$

Disclosure: Nothing to declare

\section{NP027}

Nursing-controlled adherence to treatment after ALLOSCT: Results from a pilot-study of 70 consecutives patients

Marta García Blázquez ${ }^{1,2}$, Leticia Natividad García ${ }^{\text {, }}$ Estefanía Pérez ${ }^{1,2}$, Almudena Navarro ${ }^{1}$, Ana Africa Martín $^{1}$, Salinero Peral Manuela ${ }^{1,2}$

${ }^{1}$ Hospital Clínico Universitario de Salamanca, Salamanca, Spain, 2Instituto Biosanitario de Salamanca (IBSAL), Salamanca, Spain

Background: Due to the complexity of the pharmacological treatments handled by allotransplanted patients, with an average of 12 drugs/patient and, being adherence the most important modifiable factor that influences the treatment outcome, we should provide patients with all the strategies available that allow them to take it properly. To evaluate the pharmacological adherence during the first 100 days posttransplant in a series of 70 consecutively allo-transplanted patients in our Institution. To detect, record and analyze the incidence of non-adherence to treatment.To analyze the effectiveness of the strategies used to improve adherence. 
Methods: We performed the study in two phases, the first when they attended the first post-transplant consultation after discharge and the second on day +100 post-transplant by: 1 . Objective evaluation: checking that the patient had all the prescribed medication. 2. Subjective evaluation: 2.1 Using the Morinsky-Green test that assesses the adherence reported by the patient and $2.2 \mathrm{We}$ use to value the skills and knowledge of the patient with a questionnaire adapted from the DRUGS and Med-Take scales. 3. We have recorded the factors that influence adherence in 4 sections:

Related to the patient (omission, low motivation, lack of knowledge, misunderstanding treatment instructions). Related to the treatment (complexity of the treatment, duration, frequent changes in doses, imminence of beneficial effects). Related to the disease (severity of symptoms and disease, degree of disability). Related to the health system (overload of care, short consultations, lack of review of treatment changes). 4. Recording of educational interventions, behavioral reinforcement, family / social support, techniques / skills, simplifying treatment and the combination of interventions carried out by the nurse to solve the problems detected.

\section{Results:}

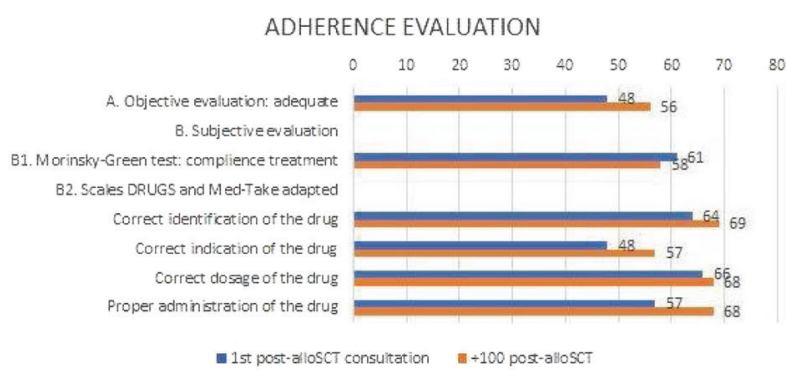

FACTORS INFLUENCING ADHERENCE
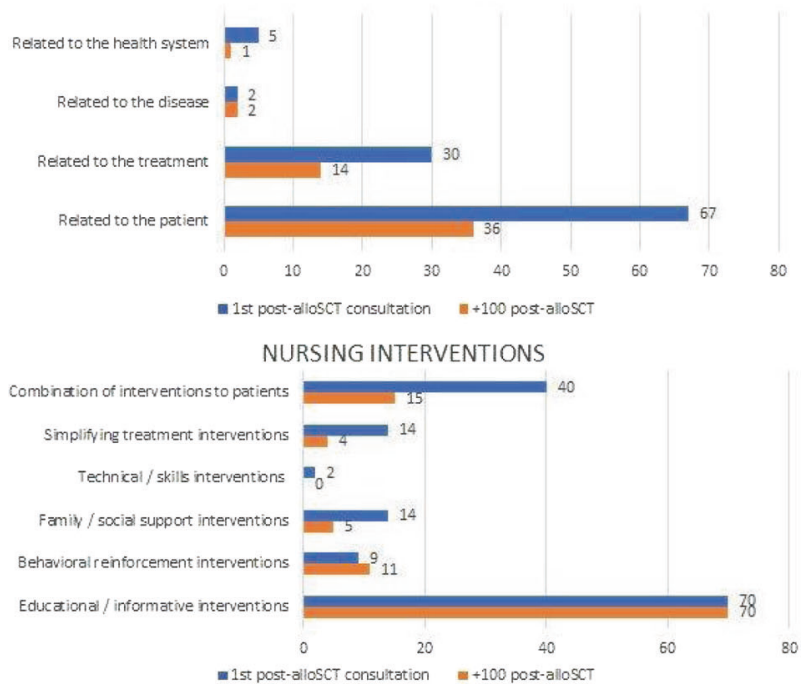

[[NP027 Image] 1. Outcomes]
Conclusions: Nursing interventions: conducting educational activities, facilitating that the hematologist simplifies the treatments and providing positive reinforcement improves the handling of the medication by the allotransplanted patients.

Disclosure: Nothing to declare.

\section{NP028 \\ Inpatient to outpatient - meeting the complex nutritional needs of the allogeneic haematopoietic stem cell transplant (HSCT) recipient following discharge}

\section{Laura Platt, Victoria Potter ${ }^{I}$, Joanna Injore ${ }^{I}$, Katie Walker $^{\text {I, Michelle Kenyon }}{ }^{I}$}

${ }^{1}$ King's College Hospital NHS Foundation Trust, London, United Kingdom

Background: Complications such as acute/chronic graft versus host disease (GvHD), recurrent infection and gastrointestinal disturbance, contribute to impaired nutritional status following HSCT (Ferreira 2014). Fifty percent of patients demonstrate malnutrition at 100 days post-HSCT, increasing morbidity and decreasing quality of life (Fuji 2015; Hung 2013). We designed an outpatient dietetic service for Allogeneic HSCT recipients to bridge the immediate post-discharge period and anticipate and meet long-term nutritional need.

Methods: The outpatient service provided 1-day per week Specialist Dietitian input. This enabled multidisciplinary meeting and clinic attendance to identify and treat at-risk patients. Nutritional assessments, risk analysis for impaired nutritional status and dietetic interventions were provided (British Dietetic Association, 2016). During the pilot phase (October-December 2017), 30 Allogeneic HSCT recipients were assessed.

Results: Recipient characteristics are summarised in Table 1 . The predominantly middle-aged cohort, averaged more than 300days post-HSCT. Most recipients had reduced intensity conditioning (RIC) and over half referred received unrelated donor transplants. No patient met criteria for being underweight pre-HSCT (NICE, 2017), while at post-HSCT assessment, mean BMI reduced by $5 \mathrm{~kg} / \mathrm{m}^{2}$ with 6 patients classified as underweight. At-risk and clinically significant percentage weight loss was observed in $27 \%$ and $70 \%$ of recipients respectively. Nutrition impact symptoms (NIS) accounted for most referrals (40\%). Nutrition counselling (NC) and supplement drinks were most common interventions. $3 / 4$ of recipients required $\geq 2$ Specialist Dietitian interventions.

Conclusions: Consistent with previous research (Hung 2013; Urbain 2012), these findings demonstrate HSCT- 
recipient deterioration in nutritional status \pm lean body mass particularly during the early post-HSCT period. The diverse referral reasons underlines the range of post-HSCT complications impacting on nutrition. Given the known association between increased nutritional risk and myeloablative conditioning (MAC) (August 2009), this cohort of 90\% RIC recipients highlights prevalence of this groups' nutritional challenges. Dietetic interventions were multifaceted with 3/ 4 recipients receiving $\geq 2$ tailored interventions. Research shows NC with or without supplement drinks is associated with significant improvement in nutrition status and outcomes (Lee 2016), illustrating the importance of individualised, patient-centred interventions. Having established need for Specialist Dietitian input, further service development will implement comprehensive nutritional screening and guidelines for Dietetic input. This pilot Outpatient Specialist Dietetic service demonstrates the complex nutritional challenges post-HSCT and the importance of a Specialist Dietetic service in supporting an MDT approach in malnutrition management and its consequences beyond the inpatient phase.

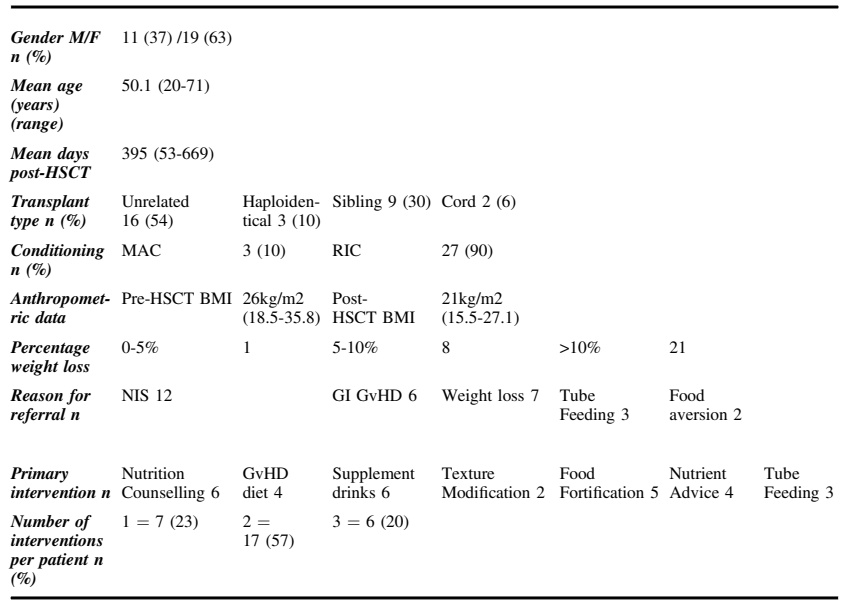

[[NP028 Table] 1. Table 1. Recipient Characteristics]

Disclosure: Nothing to Declare

\section{NP029 \\ Quality improvement based on patient perspectives on the organization of an outpatient clinic}

\section{${ }^{1}$ Lea Brandt Kristensen}

${ }^{1}$ Aarhus University Hospital, Aarhus, Denmark

Background: Patient involvement in organizational changes is a current area both politically, in health community and among health professionals. Patients achieve high treatment quality and satisfaction by being involved (Ocloo, 2016, Svederud et al., 2015, Coulter, 2012). Improvement of the organization of a transplant outpatient clinic was planned and introduced by health professionals, without considering incorporating the patient perspectives in the organization of the clinic.

The purpose of this study was to identify patient perspectives on organization of a transplant outpatient clinic to contribute with knowledge useful for further studies of patient perspectives on organization, and to discover and achieve the possibilities to use patient perspectives as a method for quality improvements.

Methods: No validated interview guide is available. A semi-structured interview guide was created based on literature review. The interview questions focused at the patient's view on possibilities of improvements in the organization of the visit in the clinic. After an actual change in the organization four semi-structured individual interviews were conducted. Patients interviewed were hematological transplant patients from an outpatient clinic at Aarhus University Hospital, Denmark. The patients were aged between 45-70 years. Analysis was conducted with a phenomenological approach using Steiner Kvale's 5 steps to interview analysis (Kvale, 2015).

Results: Three main topics were considered important to the patients in relation to the organization of the outpatient clinic: 1) The relationship between patient and health professionals, including culture and atmosphere in the outpatient clinic, to feel safe and the health professionals are well acquainted with the patient as well as the individual health problems. 2) Professional treatment including confidence in the health professionals' experiences, competencies and professional expertise. 3) Perspectives on organization including perspectives on whether the time schedule was followed, continuity, coordination and a minimization of waste time.

Discussion: Analyzing the results of the interviews clarified that patient perspectives contribute with new knowledge of parameters which patients consider important. Parameters, which differ from the organizational changes, that the health professionals focus on. The implemented organizational changes would have been different, if the patient perspective had been involved. Four patients are however too small a number to pinpoint the differences. The study was useful for getting an idea of the patient perspectives on organization for further studies, as well as knowledge of questions relevant to quality improvement focus.

Conclusions: Patients provide new perspectives on which parameters are important for the experience of treatment quality. It underpins the necessity of creating quality improvement initiatives in the healthcare system that involves patients. 
References: COULTER, A. 2012. Patient engagementwhat works? The Journal of ambulatory care management, 35, 80-89.

KVALE, S.; S. BRINKMAN (2015). InterViews: Learning the Craft of Qualitative Research Interviewing. Third edition.

OCLOO, J., MATTHEWS, R (2016). From tokenism to empowerment: progressing patient and public involvement in healthcare improvement. BMJ Qual Saf, bmjqs-2015004839.

SVEDERUD, I., et al. (2015). Patient perspectives on centralisation of low volume, highly specialised procedures in Sweden. Health Policy, 119, 1068-1075.

Disclosure: Nothing to declare

\section{NP030}

\section{Nutrional challanges in pediatric stem cell patients}

Christina Merz-Zimmara ${ }^{I}$, Lisa Manser ${ }^{I}$, Andrea Jarisch $^{1}$, Monika Beffart-Gaines ${ }^{I}$

\author{
${ }^{1}$ University Children's Hospital Frankfurt, Frankfurt, \\ Germany
}

Background: The aim of this investigation was to compare paediatric patients, who needed parenteral nutrition (PN) at home after stem cell transplantation with those who did not require this.

Methods: We compared retrospective following parameters: weight (pre- and posttransplant), retention parameter, documented oral intake, in 3 patients in each group at the age of 8-10 years in 2016-17. Patients were treated with PN when they don't reach an adequate oral intake and to reduce the stay in the hospital.

Patients having an adequate oral intake were used as control group. All patients suffered on haematological diseases.

Results: Clinical assessment of weight and intensive conversations show that PN given at home had a clear impact on the patients weight as well as their perceived general well-being. Both groups showed an essential weight loss post SCT (pre and post SCT $>2 \mathrm{~kg}$ ). The PN group gained weight quicker than the control group.

Conclusions: The generell difference between both groups was the inadequate oral intake and prescription of PN. The TPN group gained weight quicker and had a better well-being post SCT. Based on these findings we recommend that a preventative alternative, such as liquid nutrition is offered to combat weight loss after transplantation, possibly as early as the day isolation ends or towards the end of hospitalization. Why some patients generally show greater weight loss then others, cannot be established definitively.
Additional evaluations could be made by comparing a further ten patients who could take liquid nutrition versus those where this was orally not possible.

Disclosure: The authors have no conflicts to disclose

\section{NP031 \\ The role of spirituality in patients undergoing hematopoietic stem cell transplantation: A qualitative meta-synthesis}

\author{
Liyuan Zheng ${ }^{I}$, Yi Peng ${ }^{I}$ \\ ${ }^{1}$ Jilin University, Chnagchun, China
}

Background: Hematopoietic stem cell transplantation has become the standard treatment for many diseases, but it is an intense and distinctive experience for patients. During the treatment of transplantation, patients not only bear the burden of high symptoms, but also bear the psychological burden of long-term fear and hope. Spirituality is 'experiencing a meaningful connection to our core selves, others, the world, and/or a greater power, as expressed through our reflections, narratives, and actions". Studies has shown that patients need spiritual support besides physical care and psychological care and social support. Lots of studies have proved the role of spirit and belief in coping with cancer experience. It is necessary to explore the role of spiritual needs of patients undergoing hematopoietic stem cell transplantation because it may improve their quality of life and decrease mortality. However, studies rarely involved the role of spirituality in patients with hematopoietic stem cell transplantation. We conducted a meta-integration of qualitative research on the spiritual experience of patients with hematopoietic stem cell transplantation. It will also guide nurses work and provide other useful information about care strategies of patients with hematopoietic stem cell transplantation.

Methods: Searches in Pubmed, web of science, Embase, CINAHL, Cochrane Library yielded over 17 citations, which were deduplicated and then screened by two independent reviewers. Following a systematic search strategy all papers were assessed in relation to inclusion criteria and quality. English-language, primary qualitative studies that investigated the role of spiritual in patients' experiences with HSCT were included. Critical appraisal used JBI Critical Appraisal Checklist for Qualitative Research.

Results: Six primary studies were included in the metasynthesis. Twenty-four complete findings were grouped according to their similarities to form five categories. There are "believe in God and destiny", "positive view of disease", "external manifestations of spiritual coping"and "internal manifestations of spiritual coping", "spiritual dependence 
increases". These categories resulted in three synthesized findings: integration results 1: spiritual experience of illness with parents; integration results; 2 : spiritual coping style of illness with patients; integration results; 3 : spiritual changes brought about by HSCT.

Conclusions: Nurses should pay attention to spiritual experience and spiritual coping style of patients undergoing hematopoietic stem cell transplantation, and provide necessary spiritual support before and during transplantation, in order to help patients to cope with disease pressures as actively as possible, improve the quality of life of transplanted patients and reduce mortality.

Disclosure: Nothing to declare

\section{NP032}

Training of nurses in psycho-emotional issues of dying children, parents and relatives

Cornelia Duda, Andrea Jarisch ${ }^{1}$, Mario Schwarzer ${ }^{1}$, Monika Beffart-Gaines ${ }^{I}$, Peter Bader ${ }^{I}$

${ }^{1}$ University Clinic of Frankfurt, Frankfurt, Germany

Background: We take care of children and adolescents of different age as well as varying medical and nursing conditions at our stem cell transplantation center.

Although many of our patients have good prognoses after surviving stem cell transplantation, parents are concern with the question: What if not? These discussions require a lot of the staff, especially of our young and unexperienced colleagues. They do not know how to behave being confronted with questions of death, or being asked for advice. Complicating, many children express themselves in symbols or just hint their fears.

Children's understanding of death develops during life. Only with this knowledge we can assume what kind of imaginations of death they have in different ages.

It is a very individual matter of finding the right way to accompany someone dealing with his grief and could be managed better or worse by each employee. Adults with theoretical knowledge about the experiences of dying children respond more insightful to those. Maybe even adults affected by grief and death succeed better in helping children dealing with this situation loss.

Methods: We train our colleagues to help them providing advice to parents and relatives and therefore reducing anxiety and sorrow of our patients.

Results: Contents of this further education we like to provide.

Conclusions: This know-how is not only relevant at our pediatric station but especially in cases of children losing their parents, grandparents or siblings. It is essential to understand the variety of reactive behavior and imaginations of these children. But less is known about the impact of grief in children.

Disclosure: no conflict of interest

\section{NP033}

Training patient's awareness when dealing with medication during the transplantation process

Marion Simon ${ }^{1}$, Julia Ebert, Jan Grellmann ${ }^{1}$, Petra Neizel $^{I}$, Renate Herb ${ }^{I}$, Tanja Matko ${ }^{I}$, Judith Goldgruber ${ }^{I}$, Petra Wunsch ${ }^{l}$, Ute Wolf ${ }^{l}$, Claudia Barnikol-Veit ${ }^{\prime}$, Claudia Schmidt ${ }^{l}$, Ildiko Bakone-Palfi, Martina Heilmann $^{1}$, Tatiana Androuchkevitch ${ }^{I}$, Laura Blasco Bautista $^{I}$, Susanne Kramer ${ }^{I}$, Andrea Kunig ${ }^{1}$, Lisa Lesti ${ }^{1}$, Xin $\mathrm{Niu}^{1}$, Yao Qi ${ }^{I}$, Adriana Suke ${ }^{I}$, Veronika Schweighofer ${ }^{I}$, Angela Müller ${ }^{1}$

${ }^{1}$ University Hospital of Munich, München, Germany

Background: Blood and marrow transplantation (BMT) can lead to severe side effects, mostly due to the conditioning regimen, applied immunosuppressants or inappropriate medication intake throughout the process of transplantation and ambulatory aftercare of the patients, which can result in high re-admission rates.

To help BMT-survivors enjoy the best health-care possible, the BMT unit of the University Hospital of Munich, Grosshadern, LMU, offers a program to train patients' awareness when dealing with medications in the outpatient setting. The program aims to prevent and minimize drugrelated complications. Thus BMT nurses teach patients how to prepare their medication, giving instructions on drug effects and side-effects. Services are individualized, and education is verified by inpatient nurses and BMT physicians upon discharge.

Methods: All compliant patients who underwent BMT at our institution since 2016 were included. Patients with the cognitive ability to follow the instructions of the nurses and appropriate fine-motor skills were eligible. An accurate drug account has been handed over to every patient, which listed the individual medication for the day. Every patient was handed over a box with all the drug packages needed and was supplied a drug dispenser. The drug dispenser was checked once a day by the nurses' staff.

Results: Incorrect medication preparation was observed in approximately $75 \%$ of the patients at the beginning of the program. Addressing mistakes very early in the treatment course and continuous tutorial of the nurses led to a success rate of $>90 \%$ in correct drug preparation and intake when patients were discharged. Median time needed for correct drug preparation was 6 days (1-30 days). In those $<10 \%$ of 
the patients where correct drug preparation was not granted and took more than 30 days, family members or home care services were involved early for BMT patient's assistance in the outpatient sector. Up to now we included 120 patients. Main problems we see are the short-term changes in the medication plans and the respective communication.

Conclusions: In summary, the program acceptance is high and might allow to effectively minimize the drug related complications of immunosuppressants and antibiotics after BMT among survivors. We think that it constitutes a great resource and tool to help patients during inpatient stay and allows to prepare them for the outpatient setting.

Disclosure: Nothing to declare

\section{NP035}

\section{Abstract withdrawn.}

\section{NP034 \\ Experience of protective isolation in patients who have undergone hematopoietic stem cell transplantation - a survey}

\section{Sheetal Thakur', Meera Achrekar ${ }^{1}$}

${ }^{1}$ Tata Memorial Centre Advanced Centre for Treatment, Research and Education in Cancer, Navi Mumbai, India

Background: Bone marrow transplant patient are placed in protective isolation as they are immunocompromised and at risk for viral, bacterial or other opportunistic infections and also complications due to their treatment regime. Isolation can lead to feeling of loneliness, anxiety, physical problems etc. Therefore this study was undertaken to evaluate the isolation related experience of patients who were hospitalized and underwent hematopoietic stem cell transplant.

Methods: A retrospective survey was undertaken for 30 patients who have undergone hematopoietic stem cell transplant and were visiting the BMT OPD for follow up. Tools consisted of

a) five point Likert scale consisting of thirty three statements \{Part A - thirteen statements on Physical experiences and Part B - twenty statements on emotional experiences $\}$ and

b) two questions which were open ended were given to patients in a language they understand. Content validity and reliability was established. The setting was a tertiary care cancer hospital.

Results: Total 30 patients participated in this study among them $80 \%$ were males, and around $53 \%$ were single .There was equal representation (50\%) each from joint and nuclear family. Around 35\% were employed and average monthly income was above 30,000 rupees (33\%) while $27 \%$ had family income less than 5000 rupees. Majority (73\%) of the patients were diagnosed with leukaemias and around 63 $\%$ of patients had Allogenic transplant. Physical experiences during isolation: Patients experienced side effects which were bothersome $(48 \%)$ and pain $(24 \%)$ leading to inadequate sleep while $27 \%$ patients were forced to spend time in bed. Arounf $30 \%$ of patients experienced a sense of dependency as there were restrictions on ambulation and $46 \%$ had difficulty maintaining nutritional requirements due to side effects of medicines. Few patients had frequent loose stools $(24.3 \%)$ and urination $(31 \%)$ whereas some patients (27\%) had problems of dryness, itching, rashes and desquamation which caused discomfort. Emotional experiences during isolation: Around 33\% patients felt suffocated throughout their stay in the unit while $60 \%$ of patients expressed that food was not appealing as they were on bland diet. Majority (67\%) of patients expressed that their illness was a personal hardship for their family and therefore wanted to go home early after finishing the treatment (96\%). Interestingly $77 \%$ did not feel they were a burden to the family. Few of them (30\%) experienced sudden feeling of panic. Constant monitoring of health workers gave a sense of reassurance $(63 \%)$ while the diversional therapy provided in the unit helped them immensely $(70 \%)$. The major difficulties faced during bone marrow transplantation period was side effects of the treatment $(43 \%, \mathrm{n}=21)$ and feeling of loneliness (14\%) When asked for suggestions to make their experience in BMT unit better, they mentioned that good communication (12\%) and provision of health education through visual means $(6 \%)$ was required.

Conclusions: Patients undergoing hematopoietic stem cell transplant experience physical and emotional issues due to protective isolation. The nurses need to be sensitive and identify and rectify these issues in a timely manner and thus create a positive experience for the patients.

Clinical Trial Registry: Not Applicable

Disclosure: Nothing to Declare

\section{NP036}

The psycho-social effects of multiple failed transplants and long term care

\section{Elizabeth Rhodes ${ }^{1}$}

${ }^{1}$ Christie Hospital, Manchester, United Kingdom

Background: Failed transplants raise multiple psychological effects and staff having effective communication and psychosocial skills are core competencies for nurses (EBMT Handbook, 2008). 
Aplastic Anaemia (AA) fits the criteria for transplant and multiple transplants if there is engraftment failure (BSBMT, 2013).

Methods: Data relating to one case study of an AA patient has been gathered to see track the timeline of his psychological state through three failed transplants.

Case study - TYA patient, aged 18, with Severe AA had three failed transplants. First two MUD (Matched Unrelated Donor) and third a haploidentical from sibling. First diagnosed in May 2014, admitted for first transplant in July 2014 and had Fludarabine/Cyclophosphamide/CAMPATH MUD, fought sepsis, CMV reactivation and confirmed engraftment failure in August 2014.

Patient started to struggle psychologically with loss of friends and 'normal life'. He was also struggling with length of stay and prospect of further treatment and isolation. He was started on antidepressants.

Second transplant, in September 2014, was Fludarabine/ ATG RIC MUD. It bought further infections, CMV reactivation, GVHD and engraftment failure confirmed November 2014.

Patient started to 'worry for his future' and felt he had no sense of purpose. Had key worker and OT input and had good nurse-patient relationships with ward staff. Patient was eventually discharged but to come to day unit every other day, as was transfusion dependant, whilst a sibling haplo transplant was being arranged.

Admission for Fludarabine/Cyclophosphamide/TBI HAPLO in February 2015. Patient was eager to 'get on with it'. Throughout third transplant, patient's mood was low and as time passed he knew it was failing again. CMV reactivated for the third time and became life-threatening in April 2015. He improved and was discharged in May 2015 to continue with supportive care after third failed transplant. He went on living neutropenic and pancytopenic, requiring numerous hospital stays and days in the day unit having blood and platelet transfusions until he got diagnosed with AML and died in June 2018.

Results: Patient struggled greatly with the psychological side of treatment. Starting with his loss of 'normal life' and friendships, struggling with isolation and lengthy hospital stays and eventually preferring to be in hospital and becoming institutionalised, depending on nursing staff for friendships. Also nursing staff build strong nurse-patient relationships and are left feeling helpless.

Conclusions: Transplant failure can cause psychological distress and leaves patients feeling alone and uncertain about their future. Nurses and ward staff need adequate education and support to be able to support patients through long stays in hospital and isolation. MDT input was very important and has proven advantages, giving patient multiple avenues of support.
One aspect to consider is to evaluate nurses' wellbeing in regards to building bonds with patients and how to look after themselves when treatment does not work as this is recognised as one of the main stressors for staff (Futterman and Wellisch (1990)).

Disclosure: Nothing to declare

\section{NP037 \\ "Greffé \& Gourmet": Creation of a recipe book for transplant patients}

\section{Stéphanie Schmitt ${ }^{1}$, Pr. Raoul Herbrecht ${ }^{1}$}

${ }^{1}$ Hôpitaux Universitaires de Strasbourg, Strasbourg, France

Background: Allograft (and chemio /radiotherapy) causes important side effects (nausea, vomiting, mucositis), needs taking many medication, causing a loss or change of taste, weight loss, anorexia, loss of desire, depression ... Added to this, are hygiene and dietary restrictions associated with taking immunosuppressive medication leading to fragility in the face of infection. It is often an additional difficulty and loss of pleasure for patients. Or France is the country of gastronomy and great chefs! In Alsace, the pleasure of eating is a way of life, and for families, to eat is to live! Context: The "Recettes soin-plaisir" book for transplant patients published by the Limoges University Hospital (2015): patients and caregivers were very interested. Survey of interest issued to post-transplant patients in Strasbourg in 2016. Modification and alleviation of the low bacterial diet in 2016 at Strasbourg University Hospital Hence the idea to propose to the star chefs of the region to develop the recipes with the same instructions as the patients.

Methods: Goals: Regain a culinary and gastronomic pleasure with family, show that a good, beautiful, healthy and protected diet is possible, establish appetizing and creative recipes, sometimes easy, sometimes festive and rooted in a regional tradition, in spite of the food restrictions; improve the nutritional and often psychological status of patients. Steps: Research of a publishing house to elaborate and financed the book and photographer Cartoons of Gab (comic cartoonist) in order to lighten the hygiene instructions and dietary recommendations Collect recipes of the chefs Recipe photography by Karine Faby Elaboration of the book and final proofreading; preface by Pr. Herbrecht November 2, 2018: national publication. Pluriprofessional Project: Doctors of various specialties of the University Hospital, caregiver, dieticians, Alsatian chefs of Etoiles d 'Alsace; publishing house: publisher, artistic director, cartoonist, photographer, associations, patients! 
Results: Commitment and Costs: Volunteering, commitment of caregivers, of the publisher, of associations (to buy the books for our transplant patients)

Cost of the book: approx. $€ 20,000$ for 2,000 copies. Book Composition: Nutritional advice and Gab's drawings: General hygiene advice, Choice of foods and Dietary advice, pictograms and warnings. The recipes: Aperitifs and starters, Dishes: vegetarian, fish, meat and Desserts and pastries.

Thanks

Conclusions:

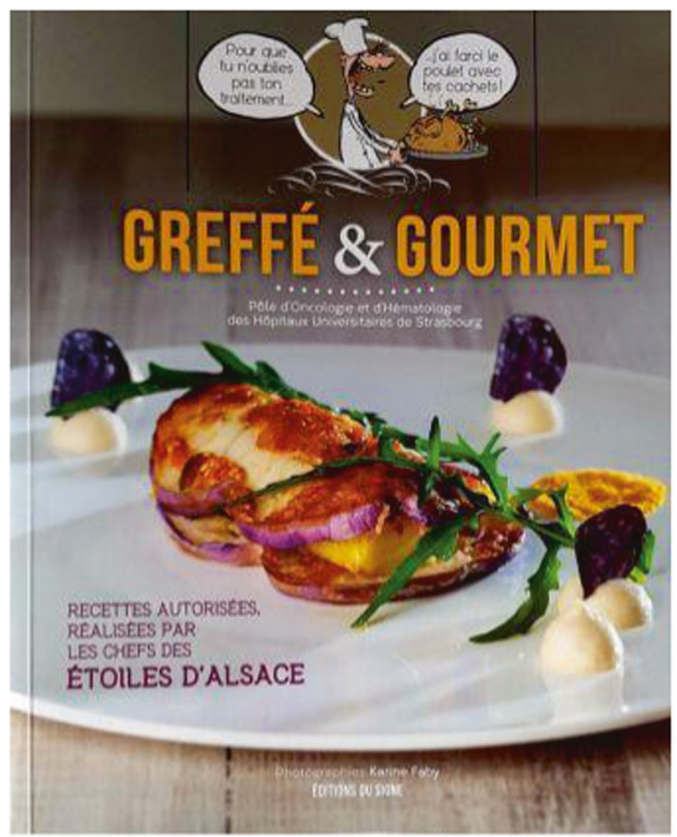

[[NP037 Image] 1. Book cover Greffé \& Gourmet]

Disclosure: Nothing to declare

\section{NP038 \\ The benefits of routine holistic needs assessment in post transplant}

\section{Lorna Welsh ${ }^{1}$}

${ }^{1}$ Queen Elizabeth University Hospital, Glasgow, United Kingdom

Background: Advances in practice for stem cell transplants has led to transplants becoming safer, the age of those being transplanted are higher and there are improvements in supportive treatments. With a growing population of transplant survivors, there is a greater need to improve posttransplant support that begins from discharge and lasts into late effects. A survey of HSCT recipients in 2016 found that only half of patients that needed emotional or psychological support and practical support like getting back to work actually received it. And 1 in 3 patients did not receive information they wanted about support (Anthony Nolan, Recovery After Transplant: Who Cares? 2017) Horne, B et al (2013).

In 2017 Anthony Nolan began funding a post transplant clinical nurse specialist at the Queen Elizabeth University Hospital, Glasgow. This poster aims to look at the impact of routine holistic needs assessment post-transplant by the CNS over a 14 month time period.

Methods: Introduce a Holistic Needs Assessment (HNA) and Care Plan when discharged up to 1 month, Assess patients at Day 100 reviewing Appetite/Nutrition, Physical Exercise/Fatigue, Psychological (performing PHQ4),Memory/Concentration, Sexual Function, Work/ Finances, Performance Status (Karnofsky Score) and Assess patients at 6 months and 1 year using local Recommended Screening and Preventative Practices for Adult Long-Term Survivors after Allogeneic Haemopoietic Stem Cell Transplant (HCT)

Results: A total of 70 HNA and Care Plans were conducted. Results from HNA are as chart below:

From the Care Plans developed: 19 referrals were made to: Dietician, Physiotherapist, Psychologist and Occupational Therapist; 14 written supportive information booklets were given; 5 people were signposted to local benefit teams; 2 Anthony Nolan Grants applications; 1 referral to local services; 1 given information on local support centre; 1 given details of mindfulness app. At Day 100 A total of 60 assessments were conducted. As a result: 20 referrals were made (physiotherapist, Dietician, Psychologist, Wig referral, Gynaecologist); 50 written supportive information booklet were given to patients, 12 Anthony Nolan grants applications and 7 patients were signposted to benefit teams. At 6 Months A total of 23 Assessments were conducted. From this: 2 Anthony Nolan grants applications, 1 referral to local benefit team and 9 written supportive information booklets given. At 1 year A total of 20 assessments were conducted. From this 1 patient received written supportive information booklet, 1 referral was made and 2 patients identified as having high cholesterol and 1 dexa scan conducted as part of recommended follow up. The introduction of the 6 month and 1 year assessment is more recent therefore numbers are lower at this stage.

Conclusions: The findings demonstrate the need for early identification and referral to existing support services. This can be achieved from routine assessments from a CNS. Although patients would still be referred without these assessments there would be no way of tracking the referrals or outcomes. The data supports the need for a variation in the provision of support including written information. The use of routine holistic needs assessments provides 
consistency in practice and encourages the inclusion of other support services.

Disclosure: Nothing to declare

\section{NP039}

Delivering autologous peripheral blood stem cell transplant in an ambulatory care setting; patient and service perspective

\section{Deirdre Young ${ }^{1}$, Yvonne Patefield-Smith ${ }^{1}$, Rebecca Hadrill $^{l}$, Joanna Wyatt ${ }^{1}$, Rachel Miller ${ }^{I}$}

${ }^{1}$ Oxford University Hospitals NHS Foundation Trust, Oxford, United Kingdom

Background: The World Health Organisation (WHO) (2003) have spoken out on the current global status of Cancer affirming, "The worldwide burden of Cancer warrants more efficient and effective cancer care management."

In order to manage the growing demand locally on our own Bone Marrow Transplant Service we have considered alternative options for standard, inpatient care delivery. This thinking encompassed a parallel aim to positively impact patient outcomes, influence inpatient flow and reduce the inpatient stay for Autologous PBSCT. National Institute of Clinical Excellence guidelines (NICE) (2016) advocate the consideration of Ambulatory Care (AC) for adults and young people with haematological malignancies. We chose 6 patients who met these criteria and others, ensuring AC was a treatment option that reflected what was important to them (NICE 2012). The Cancer Reform Strategy (2007) provide valuable evidence to support an Ambulatory model of care in striving to improve patient experience, whilst increasing efficiency of services. AC is a safe alternative to inpatient care in many instances and frees up beds for those patients requiring them (Sive et. al 2012).

Methods: Our Ambulatory Nursing team have collected data from 6 patients treated with Autologous PBSCT between October 2017 and November 2018. The collection comprised of qualitative data obtained through patient evaluation of the service at the end of their treatment and analysis of usual inpatient stay for such treatment versus nights saved through delivering care in the AC setting. This allows us to predict the yearly overnight stays that could be prevented through an AC Unit, caring for approximately 6 Autologous PBSCT patients a month.

Results: Patient evaluation of the service has been hugely positive. Themes emerging around the benefits of being able to sleep comfortably at home and choosing "what I eat and when," are suggestive that high dose treatment is something that can fit into the routine of normal life in the absence of the need for inpatient intervention.
One patient claims that the experience has been "invaluable" in allowing normalcy to continue for her children whilst she undergoes her BEAM Autograft. This has had a hugely beneficial impact on her psychological well being as her children's welfare was a primary concern.

Data collection of the 3 patients treated with BEAM Autograft concludes that 21 overnight stays were avoided through Ambulatory delivery. The other 3 patients treated with HD Melphalan Autograft allowed us to reduce demand on the inpatient setting by 20 overnight stays.

Conclusions: We can predict that establishing $\mathrm{AC}$ as a standard delivery model for Autografts in patients who meet exclusive criteria could reduce reliance on inpatient beds by 492 nights per year (based on our capacity as a specialist nursing team to treat 6 Autograft patients per month.) This could see inpatient transplantation activity increase as well as overall productivity of the BMT service. We can also better appreciate the positive outcomes for patients whose preference it is to remain at home where possible, and the importance of their individual needs informing the decision making process regarding where their care should be delivered.

Disclosure: Nothing to declare

\section{NP040}

\section{Abstract withdrawn}

\section{NP041}

Role of nursing staff in the implementation of medication training of patients from the day of admission

\section{Stefanie Yilmaz ${ }^{1}$}

${ }^{1}$ Universitätsklinikum Frankfurt, Frankfurt am Main, Germany

Background: During their stay in the hospital and after discharge, patients had more and more difficulties with the prescribed medication and made mistakes in the preparation and intake of their respective drugs. Especially for immunosuppressive drugs it is vital to adhere to the prescribed schedule and doses.

Methods: On the day of admission to the ward, the oral medication is specified in the digital patient file by the treating physician. During the afternoon, the responsible nursing staff will print out an updated medication schedule for the respective patient which is valid for the next day. They will also prepare a plastic tray containing, a blister of each drug prescribed and two drug dispensers. The patient will be informed by the nursing staff as well as the medical team about the indication of each drug, the exact dosage and 
the application schedule. During the afternoon, the patient will assort his medication by filling each tablet into the respective box of the dispenser, which is monitored by the nursing staff. If the patient has repeatedly taken his medication correctly, he will no longer be monitored and is allowed to assort his medication independently. However, he will be reminded daily in each shift to take and prepare the medication, if necessary. Changes of the oral medication in the course of the day will prompt printing and hand-out of an updated medication schedule to the patient. After discharge, the drug training project will be continued in our outpatient clinic.

Results: The intake of the medication was more reliable and self-dependent. The compliance with the medication regimen, as well as the quality of life and the sense of responsibility were enhanced. For the patients who were not able to adhere to the medication regimen, the family or a social service could be involved at an early stage. In that case, the medication was assorted during the hospital stay by the responsible nurse in the ward room.

Conclusions: We found the medication training in our patients to be extremely beneficial, as confirmed by the patients. We observed less medication errors and patients grew more confident in their ability to prepare and administer their medication. After discharge, they will have to take about 30 tablets/ day. Maintaining the 12-hour interval between each dosage of immunosuppressiv medication and ensuring that medication was taken after the blood collection (important for proper control of trough levels in the peripheral blood) was no longer an issue.

\section{Literature:}

1. Levine AM, Richardson JL, Marks G, Chan K, Graham J, Selser JN, Kishbaugh C, Shelton CR, and Anderso Johnson C; "Compliance with oral drug therapy in patients with hematologic malignancy";

Journal of clinical oncology, Vol 5,No 9 (September),1987:pp1469-1476

2. Asani F, Kissling W, Pitschel- Walz G., Bäuml J.; "Medikamententraining"; Psych Pflege 2006,12:205-207

Clinical Trial Registry: nothing applicable

Disclosure: Nothing to disclose.

\section{NP042}

Anxiety and depression of adult patients before transplantation of human hematopoietic stem cells

\author{
Xia Yan ${ }^{1}$, Ting Wang ${ }^{1}$, Wei Hu ${ }^{1}$, Hua Jiang ${ }^{2}$, Xiaodong \\ $X u^{1}$ \\ ${ }^{1}$ Peking University People's Hospital, Beijing, China, ${ }^{2}$ Pek- \\ ing University Health Science Center, Beijing, China
}

Background: To investigate the anxiety and depression of patients before hematopoietic stem cell transplantation and explore their influencing factors.

Methods: The cluster sampling method was applied from May 2017 to March 2018 in one of the tertiary hospitals in Beijing and 408 adult patients were recruited. Selfdesigned questionnaires, Hospital Anxiety and Depression Scale (HADS), Functional Assessment Scale for Bone Marrow Transplantation (FACT-BMT), Functional Assessment of Chronic Diseases-Fatigue Scale (FACITFatigue), and Family adaptation, partnership, growth, affection, resolve (APGAR) were used.

Results: Anxiety status: Negative 127 (31.6\%), Suspected 198 (49.3\%), Positive 77 (19.2\%); Depression status: Negative 245 (60.9\%), Suspected 98 (24.4\%), Positive 59 $(14.7 \%)$. The binary logistic results indicated that family function is a positive influential factor for anxiety and depression in patients before hematopoietic stem cell transplantation.

Conclusions: The prevalence of anxiety and depression in patients before hematopoietic stem cell transplantation should be paid much attention to. The purposive psychological intervention should be implemented to the adult patients with consideration of the positive influence of family function.

Disclosure: Nothing to declare

\section{NP043}

Changes in nutritional status in patients on peripheral stem cell transplantation

Ljiljana Pomper $^{1}$, Irena Vidovic ${ }^{1}$, Ruza Jakovac ${ }^{1}$

${ }^{1}$ Clinical Hospital Merkur, Zagreb, Croatia

Background: PBSCT is a procedure that increases the risk of malnutrition of hematologic patients, which may be associated with multiple complications and poorer quality of life for patients during and after the procedure. Identification of patients with nutritional risk and monitoring of nutritional status changes allows timely interventions to prevent further deterioration and improve outcomes.

Methods: From 1 April to 30 September 2018, a prospective study was performed on 39 consecutive patients treated by PBSCT procedure, who were in protective isolation rooms. There were 21 men and 18 women. Median age was 62 (22-68), 26 patients were with Multiple Myeloma, 11 NHL patients, and 2 patients with Hodgkin's lymphoma. The conditioning protocols were high doses of melphalan (26 patients with myeloma) and BEAM (13 patients with NHL). Nutritional status was assessed using the Risk Assessment Form NRS 2002 at the beginning of 
hospitalization, discharge and post-release examinations, and for subtle physical changes the Tanita MC-780 scale was used.

Results: The median of hospitalization duration was 18 days. Most of the patients (92.3\%) had NRS 20023 points at start. According to BMI 13 (33.3\%) it was normal, $17(43.6 \%)$ had excessive body weight, $7(17.9 \%)$ was obese, $1(2.56 \%)$ was extremely obese and only one patient $(2.56 \%)$ was malnourished. At the end of hospitalization, BMI was underweight for 2 patients (5.12\%). Only 3 patients $(7.69 \%)$ at the beginning of hospitalization had less energy intake than needed. Only 4 patients $(10.29 \%)$ lost their body weight back 3 months. During hospitalization, 37 patients $(94.87 \%)$ lost body weight, while 2 patients $(5.12 \%)$ increased body weight of less then $1 \%$. Body weight loss was significant ( $\geq 5 \%$ body weight) in 16 patients $(41,02 \%)$, of which only 1 patient lost> $10 \%$. According to NRS 2002, 18 patients were undernourished during at the end of hospitalization (BMI $<20.5 \%$, food intake $<60 \%$, weight loss $>5 \%$ ). Food interventions were performed in all patients, while the enteral composition was taken by only 3 patients at the time of admission, 2 during hospitalization and 2 at discharge, and parenteral diet during hospitalization by 1 patient. The changes in evaluated parameters are shown in Table 1, showing base-line and end evaluation, quantification of changes and statistical significance in paired t-test. In addition, those parameters although consistently well correlate among themselves also cover different aspects of nutritional impairment and thus enable more complete evaluation of complex clinical problem.

Conclusions: The nutrition status can be more precisely evaluated than is usually done. The results obtained by the presented evaluation panel are discrete, but statistically significant and consistent. This supports the wider application of the analyzed parameters panel in routine clinical practice. The process of evaluating the nutritional status can provide credible and unbiased estimates of discrete nutritional status and provide the basis for a rational approach to their correction.

Disclosure: No conflict of interest.

\section{NP044}

\section{Patient satisfaction with nursing care received in} hematopoietic stem cell transplant unit: A survey

\author{
Suman Kubal', Meera Achrekar ${ }^{I}$ \\ ${ }^{1}$ Tata Memorial Centre Advanced Centre for Treatment, \\ Research and Education in Cancer, Navi Mumbai, India
}

Background: Patient satisfaction though an important indicator of quality care is multifaceted and challenging outcome to define. Patients are vulnerable more so when they are diagnosed with cancer and admitted to bone marrow transplant unit. Patients stay is also prolonged due to transplant related complications. Nurses care for patients round the clock and therefore it is essential that patients are satisfied with the nursing care provided to them.

Methods: A retrospective study was undertaken for 15 patients who have completed hematopoietic stem cell transplant from April 2018 - September 2018. Non probability convenient sampling was used A five point Likert scale with 25 statement was administered to patients. Patients who had completed their treatment, were over 18 years of age and willing to participate in the study and were available during the study period were included. Content validity and Reliability was established. Data was analysed using descriptive /inferential statistics.

Results: The median age of patients was 38 years. Most (87\%) of the participants were males, and $60 \%$ were married. Around $73 \%$ were diagnosed with Leukaemia's and had undergone allogeneic stem cell transplant. The complications included neutropenic sepsis (93\%), mucositis (73\%), diarrhoea (80\%). Patients were satisfied (100\%) with knowledge of nurses while $97 \%$ of patients expressed satisfaction with nurses skill and for providing a conducive environment. Two areas which required enforcements were that nurses spend time talking to them (27\%), inform them of physicians orders $(20 \%)$. There was no association between responses and demographic variables. When asked to rate overall satisfaction with nursing care, around $6.7 \%$ were satisfied and $93.3 \%$ were strongly satisfied with nursing care received in Hematopoietic Stem Cell Transplant Unit.

Conclusions: Patients were satisfied with nursing care provided during their stay in the Hematopoietic Stem Cell Transplant Unit, though there is scope for improvement. Nurses need to spend quality time talking to patient and involve patient in care related informations.

Clinical Trial Registry: Not applicable

Disclosure: Nothing to Declare

\section{NP045}

Abstract already published.

NP046

A pilot study of oral mucositis among childhood cancer therapy in yangon children hospital, Myanmar

Su Myat ${ }^{1}$, Nant Win Kyi ${ }^{1}$, Tint Myo Hnin ${ }^{2}$ 
${ }^{1}$ University of Nursing Yangon, Yangon, Myanmar, ${ }^{2}$ Yangon Children Hospital, Yangon, Myanmar

Background: Chemotherapy induced oral mucositis (CIOM) is one of the common manifestations and can cause pain, infections, delay in chemotherapy and restriction of oral intake leading to malnutrition. As a result, the CIOM is a common factor leading to increase morbidity and mortality in anticancer treatment. Foreseeing the factors leading to the $\mathrm{OM}$, and taking the common precautions may reduce the incidence of $\mathrm{OM}$.

Methods: This study is a retrospective chart review of the cases of $\mathrm{OM}$ admitted to Yangon Children Hospital (YCH), Hematology-oncology Unit from September 2017 to October 2018.

Results: Altogether 65 children were collected with M:F $1.5: 1$ but this is not statistically significant $P$-value 0.34 . The age ranged from 2-10 years with mean age 5.1. Among the grading of oral mucositis according to WHO grading, grade $3 \mathrm{OM}$ is highest, $40(61.5 \%)$ and the grade1,2,4 were $4(6.2 \%), 14(21.5 \%), 7(10.8 \%)$ respectively. During the study period, the proportion of mucositis was $2.0 \%$ and monthly proportion ranging from $0.6 \%$ to $6.7 \%$. Regarding the diagnosis of the oral mucositis children, the ALL was 55 (84.6\%), AML was 5(7.7\%), NHL was $4(6.2 \%)$ and osteosarcoma $1(1.5 \%)$. The distribution of drug treatment given among the study population with oral mucositis were cappizi methotrexate $39(60 \%)$, high dose methotrexate 5 $(7.7 \%)$, oral methotrexate $1(1.5 \%)$, IT methotrexate 3 (4.6\%), anthracycline alone $4(6.2 \%)$, anthracycline plus IT methotrexate $11(16.2 \%)$ and severe neutropenia $2(3.1 \%)$. In review of the treatment received for OM, only grade 4 patients need oral hydration and 4 patients need IV antibiotics.

Conclusions: This pilot retrospective review of the children with cancer receiving chemotherapy revealed a significant proportion of oral mucositis. A prospective study with a larger study group accounting the nutritional status the duration of treatment, interruption of chemotherapy and outcome measure should be done so as to measure the risk factors and preventive measures to reduce the incidence of $\mathrm{OM}$ in children with cancer in $\mathrm{YCH}$.

Disclosure: I have nothing to disclose about this study and have no conflict of interest.

\section{NP047 \\ Using peripherally inserted catheters (PICC) to infuse stem cells}

Angus Wilson ${ }^{1}$, Deborah Anderson ${ }^{2}$, Lawrence Daniel ${ }^{2}$
${ }^{1}$ Barts NHS Trust, Ward 5D Cancer Services, London, United Kingdom, ${ }^{2}$ Barts NHS Trust, London, United Kingdom

Background: PICC lines have traditionally not be considered appropriate for administration of stem cells in our clinical setting resulting in the patient having to have a peripheral cannula when a large central venous catheter is not present, this can bring extra distress to patients. Although the reason for not using PICC lines is not well documented there was concern that thinner lumen and presence of a valve could damage the integrity of the stem cells. Another concern is the higher rates of phlebitis associated with PICCs versus larger central lines (Harter et al,2003) further exacerbated by the high molarity of DMSO $(1.42 \mathrm{~mol} / \mathrm{L})$, which may also cause painful phlebitis (Sauer-Heilborn, Kadidlo and McCullough, 2004).

In 2015, Barts Cancer Centre began using power PICC Lines rather than standard PICCs. These lines have a power Injectable mechanism allowing for higher flow rates. It was thought that staff could use these lines for the infusion of stem cells as they have a wider bore and reduce patient distress.

Methods: All patients undergoing an autologous transplant who normally receive a peripheral cannula were included in an audit of using the PICC line for stem cell infusions. Only cryopreserves cells were included as these were the infusions taking a longer period of time. Cryopreserved cells are administered as a bolus by the nursing team allowing any adverse incidents with the PICC line to be detected promptly. Data was then collected over a 13month period from 1st July 2016 to August 2017.

Results: The audit had 42 cryopreserved cells and 4 fresh cells. The median time the PICC line was in situ prior to stem cell infusion was 3 days most frequently in for 2 days. No correlation was found between the time the line was in situ to incidents or engraftment times. No correlation was found between reported incidents and engraftment times, median neutrophil engraftment occurred on D+10 this is in line with our Trust data and British Society of Bone Marrow Transplantation data. Median Platelet engraftment $(\geq 20 \times 109 / 1)$ occurred on $D+13$. This is in line with our Trust data Median Platelet engraftment $(\geq 50 \mathrm{x}$ 109/l) occurred on D+16. This is line with our Trust data.

Conclusions: The use of power PICCs to re-infuse stem cells had no detrimental affect on engraftment times. The patients' feedback was positive compared to undergoing peripheral cannulation and the risk of a malfunctioning cannula. Power PICC lines can be used to infuse stem cells, further monitoring is required if PICCs are to be used for the infusion of non-cryopreserved stem cells.

Disclosure: Nothing to declare 


\section{NP048}

Improving of safety practice in administration and management of chemotherapy in paediatric haematopoietic stem cell transplantation

Corline Kuijt $^{I}$, Marjolijn Dogterom ${ }^{1}$, Saskia Juffermans ${ }^{1}$, Maria de Taeye-Veldhuijzen ${ }^{1}$, Heidi Verweij-Baars ${ }^{1}$, Arjan Lankester ${ }^{1}$, Hilda Mekelenkamp ${ }^{1}$

${ }^{1}$ Leiden University Medical Center, Leiden, Netherlands

Background: The preparative regimen for haematopoietic stem cell transplantation (HSCT) patients includes administration of chemotherapy. Management and administration of chemotherapy should be performed in a safe way, according to institutional and national regulations. Therefore a process of continuous learning was initiated with the aim to improve safety practices in administration and management of chemotherapy on our paediatric stem cell transplantation ward.

Methods: The administration and management of chemotherapy by nurses was evaluated with use of a questionnaire, audits and swap tests. The questionnaire was based on topics of the institutional standards and was carried out with use of an online quiz. Nurses of the working group chemotherapy audited the administration and management of chemotherapy. With use of swap tests, different parts of the ward were tested on contamination of chemotherapy. Subsequently, improvements based on the outcomes were implemented, followed by a second evaluation.

Results: During the first evaluation in Q1 of 2018 (T0), twenty-seven nurses participated in the quiz and five administration procedures were audited. Eighteen parts of the ward were tested on chemotherapy contamination in 2017.

Some practical improvements were implemented, like putting spill sets in every patient room, the patient information on handling chemotherapy was improved and room cleaning was adjusted. These interventions were implemented by communication in the weekly newsletter, during theoretical trainings and by bed-side teaching.

The second evaluation took place during Q3 of 2018 (T1). Eight nurses participated in the questionnaire and six administration procedures were audited. The results of the evaluation rounds are shown in table one. The results of the swap tests from 2018 are pending.

The chemotherapy swap tests showed that seven out of eighteen parts of the ward were contaminated with one type of chemotherapy (39\%). Only one test with exceeded the testing level and the other tests were below testing level.

Conclusions: The results of the questionnaire showed that nurses expected they had higher levels of knowledge about administration and management of chemotherapy compared to what they showed in practice during the audits. An exception was handling contaminated materials, which was good performed in practice, compared to weaker knowledge in the questionnaire. The good results of the swap tests confirmed the good performance of handling contaminated materials. The lack of knowledge in the pretest is probably related to specific calamity rules, which were questioned in the quiz, but were not so common in daily practice. Therefore practical calamity trainings on chemotherapy exposure were organised.

Based on the results we conclude that the paediatric HSCT department works safely with chemotherapy. The use of several methods for evaluating the administration and management of chemotherapy provided a useful basis for reducing risks of chemotherapy exposure and thereby improving the safety in daily practice. Further future improvements can be made based on new evaluations.

Disclosure: No conflict of interest.

NP049

relevance of the NANDA I diagnosis in patients undergoing haematopoietic stem cell transplantation (HSCT)

\section{Marco Cioce ${ }^{1}$, Stefano Botti ${ }^{2}$, Laura Orlando ${ }^{3}$, Gianpaolo Gargiulo $^{4}$}

${ }^{1}$ IRCC Gemelli polyclinic, Roma, Italy, ${ }^{2}$ Health Authority USL-IRCCS, Reggio Emilia, Italy, ${ }^{3}$ IEO European Oncologic Institute, Milan, Italy, ${ }^{4}$ Federico II University Polyclinic, Napoli, Italy

Background: Haematopoietic stem cell transplantation (HSCT) has become the standard of care for many haematological diseases unresponsive to traditional treatments; pretransplant therapy still causes high mortality and morbidity today. Due to the high risk, patient care requires careful assessment and often complex, intensive and nonrisk free interventions; the NANDA-I classification provides a way to classify and standardise areas of nursing interest. In 2011, Speksnijder, using the Delphi technique, identified 68 diagnoses relevant to identify health problems in the field of oncohaematology.

Objective: Based on Speksnijder's contribution, the study tries to identify which of these diagnoses were relevant in identifying health problems in HSCT patients.

Methods: A quantitative-descriptive study was carried out; a structured questionnaire on a 4-point Likert scale was used to build consensus, involving 57 expert nurses enrolled in the Italian GITMO Centres.

Results: Of the 68 NANDA-I diagnoses defined as relevant by Speksnijder in the oncohaematological field, this study identified 37 as relevant in detecting health problems in patients undergoing HSCT (34 in Round 1, 3 in 
Round 2) and also defined 12 as important diagnoses having obtained an absolute consensus.

Conclusions: Using the NANDA-I diagnoses to identify, from a panel of experts, the most important and relevant health problems associated with the toxicity of pre-TCSE treatment, allows to focus attention on expected and known outcomes in order to be able to intervene with performances aimed at reducing specific risks and complications.

Using NANDA-I diagnoses to know, from a panel of experts, the most important and relevant health problems associated with the toxicity of the pre-HSCT treatment, allows to focus on the expected and known outcomes, so it can possibile to intervene with performance aimed at reducing risks and specific complications. The planning of the interventions using this system, integrated with the 11 functional models of Gordon, would allow the clinician to face in a consistent, systematic and timely manner the problems of the person in this care setting, accompanying him in the decision making for the achievement of single health objectives. An accurate and valid nursing diagnosis guides the selection of health outcomes, sensitive to nursing care (Nursing Outcome classification) and the identification of interventions and activities (Nursing Intervention Classification) able to produce desired and shared results.

Disclosure: there is no conflict of interest

\section{NP050}

\section{Nutritional support practices of hematopoietic stem cell transplantation centers in China Mainland}

\author{
Yun Fang ${ }^{1}$, Minjie Liu ${ }^{1}$ \\ ${ }^{1}$ Huazhong University of Science and Technology, Wuhan, \\ China
}

Background: As malnutrition is associated with increased mortality and morbidity, poor quality of life (QOL), and prolonged length of hospital stay in patients with hematopoietic stem cell transplantation (HSCT), nutritional intervention is highly important. Herein, the aim of the study was to better understand differences between clinical practices and international recommendations as well as possible barriers to the use of nutritional therapy in patients undergoing HSCT.

Methods: An evidenced based nutrition support pathway for HSCT patients were conducted through a systematic literature review, to identify evidence and recommendations relating to the issues. Then a questionnaire focused on 4 topics (assessment and screening for malnutrition, neutropenic diet, nutritional support therapy, nutrition support in GVHD) was developed by the study authors and used for data collection. The questionnaire was placed onto a web platform, and an invitation email with a link to the survey was sent for those who had not responded.
Results: General assessment and/or screening for malnutrition of HSCT patients was performed at $72 \%(13 / 18)$ of centers. Nutritional screening tools or methods were various among the centers, and mainly used the validated nutritional risk score (NRS 2002). As many as 72\% (13/18) of centers considered the neutropenic diet (ND) as one part of the management for HSCT patients, but only one center has a formal ND protocol in place for transplant recipients. Oral nutritional supplements were used in 61\% (11/18) centers. PN has been given as the first option by the majority of centers to HSCT patients, in spite of the current guideline recommendations that favor EN over PN. Criteria for starting nutritional support in patients with GI GVHD were heterogeneous in these centers. Where NS therapy was necessary for patients with DT-GVHD, PN being the most widely used technique $(94 \%, 17 / 18)$.

Conclusions: The survey result revealed significant heterogeneity with regard to nutritional support practices among the centers, as well as practices with the guidelines. Randomized and controlled studies (RCTs) addressing these issues are also needed.

Clinical Trial Registry: no

Disclosure: Nothing to declare

\section{NP051}

\section{Understanding of nursing practices in Turkey} hematopoietic stem cell transplantation centers

Seckin Erdal', Serife Kocubaba ${ }^{2}$, Nevin Cetin ${ }^{3}$, Fatma Tekin $^{3}$, Fatma Bilgin', Merve Harman', Esra Bayrak', Gulbeyaz $\mathrm{Can}^{7}$

${ }^{1}$ Acibadem Altunizade Hospital, Istanbul, Turkey, ${ }^{2}$ Memorial Bahcelievler Hospital, Turkey, ${ }^{3}$ Hacettepe University Hospital, Ankara, Turkey, ${ }^{4}$ IU Capa Tip Faculty Hospital, Istanbul, Turkey, ${ }^{5}$ Altınbas University, Istanbul, Turkey, ${ }^{6}$ ASM Hospital, Istanbul, Turkey, ${ }^{7}$ IU Florence Nightingale Nursing Faculty, Istanbul, Turkey

Background: Hematopoietic stem cell transplantation is a challenging treatment process which patients and health workers had to cope with many problems. The standardization of patient care based on clinical study results are important in order to improve the quality of nursing care, patient safety and patient satisfaction. This study was carried out to define nursing care practices in children and adult bone marrow transplantation (BMT) units in order to determine the discussion topics that will be forming the decision voting in the 2018 Hematopoietic Stem Cell Transplant Consensus Meeting.

Methods: In this descriptive study, all of the children and adult patients BMT Units in Turkey were invited on- 
line. Out of 87 centers in our country, $69(86,25 \%)$ approved to participate in the study. 4 centers were not opened yet, 3 centers were closed and 11 centers refused to participate in this study. Using an on-line evaluation form prepared by the researchers, the clinical nursing practices were determined under 5 main topics such as Mucositis Prevention and Care, Catheter Care, Neutropenic Diet Use, Protective Isolation and Skin Care. Descriptive statistics and comparison analysis were used in the statistical evaluation of the data performed in the SPSS program.

Results: The study included 69 units in 51 hospitals in different regions of Turkey. From the centers that were included in the study, $68.1 \%$ were from adult bone marrow transplantation units and $31.9 \%$ were children's bone marrow transplantation units.

Patients in most of the centers had routine dental examination before transplantation $(82,6 \%)$ and frequency of the oral care is planning depending on the grade of oral mucositis $(88,4 \%)$.

Skin care practices vary from unit to unit. $29 \%$ use Chlorhexidine during bathing. Some of the centers recommend GvHH patients to have daily shower (33.3\%) and use oil-based cosmetics for skin care $(27,5 \%)$.

Catheter care practices also vary from unit to unit. $60.9 \%$ units' changes the cathether sponge dressing every other day. $75,4 \%$ centers change Tegaderm dressing once a week. $65.2 \%$ of the units were heparinized catheter line by preparing heparin as $100 \mathrm{U} / \mathrm{ml}$.

$95.7 \%$ of the centers use neutropenic diet. The standards of neutropenic diet such as its ingredients has not been determined in these units.

Healthcare professional in $94.2 \%$ of units wear a 3-coat standard mask, $40.6 \%$ wear aprons, $18.8 \%$ wear galoshes and $21.7 \%$ wear a bone when entering in neutropenic patient's room. The visitors of the patient in $63,8 \%$ units wear an apron and in $47,8 \%$ wear galoshes when entering in neutropenic patient's room.

Conclusions: This study results shown that the BMT care practices vary from one unit to unit in Turkey. By using evidence based nursing approach, the patient care standards and protocols should be established to provide quality and safely care for patients during BMT in our country.

Disclosure: Nothing to declare

\section{NP052 \\ Swimming against the tide of carbapenem resistant enterobacteriacae: Our challenges in clinical haematology}

\section{Joy Antonette Pe Benito ${ }^{I}$, Frances Sarkari, Karen Bradley $^{1}$}

Hammersmith Hospital, London, United Kingdom

Background: Carbapenemase Producing Enterobacteriaceae (CPE) is a large family of bacteria that usually live harmlessly in the gut of all humans and animals. These organisms are some of the most common causes of urinary tract, intra-abdominal and blood stream infections. Worldwide, a small but increasing number of strains of enterobacteriaceae have become resistant to carbapenem antibiotics. Carbapenem has been defined by the World Health Organisation (WHO) as critically important antibiotics. Clinical Haematology department at Imperial College healthcare NHS Trust (ICHNT) is extremely challenged with the emergence of CPE due to the potential risks factors that it presents to the patient population. These groups of patients are at high risk of becoming contaminated with CPE due to significant immuno-suppression. The strains tend to emerge among the group of patients where antibiotic usage is high.

Methods: The Department's first confirmed case of CPE was reported in one of the in-patient transplant wards in September 2015, which was quickly followed by five patients identified. This led to patients' screening on admission and weekly thereafter. The screening programme initiative increased awareness of the prevalence of CPE in the community. Patients were also nursed in single rooms. Moreover, staffs were re-assessed on ANTT with emphasis on strict adherence to infection and prevention control (IPC) practices. The nursing and medical team worked collaboratively with IPC, Estates and Facilities and Domestics to identify the cause of transmission with focus on preventing its spread.

The bigger challenge is that our haematology patients will have multiple presentations into one of the in-patient wards or into the day care setting where there is limited scope to isolation facilities. Thus, in 2017 as evidenced by the data collected by the IPC team, there were further two recorded incidents of CPE cross contamination between patients in the unit as confirmed by typing four patients in total. It was also collated that from January to May 2018, there were nine patients identified from the three inpatient areas.

Results: The exact route of transmission is uncertain. Whilst there is a risk associated with IPC practices, there are still significant environmental issues related to cleaning and estates that cannot be discounted as contributory factors. Enhanced chlorine and hydrogen peroxide vapour (HPV) cleaning of wards were implemented however; CPE eradication from the environment remains a challenge

Conclusions: The management of CPE within haematology remains a challenge, and requires continued multidisciplinary approach. High sample quality and its likelihood of detecting a CPE positive case can also vary 
between practitioners. Nursing education on technique has continued throughout. Resource limitations have prevented other centres from implementing a rigorous screening process such as ICHNT. The demand of resources and isolation facilities is only set to increase in future.

CPE continues to pose a serious threat to our vulnerable patients. Prevention and zero transmission of CPE is our key focus for the future management; for how long we can with this increasingly world-wide problem remains unclear

Clinical Trial Registry: Not applicable

Disclosure: No conflict of interest

\section{NP053}

Impact of weekly stem cell coordination round on pediatric transplant activities: Real - world experience from a tertiary care hospital in Kingdom of Saudi Arabia

Donya Qattan', Amal Alseraihy ${ }^{1}$, Mouhab Ayas ${ }^{1}$, Ibrahim

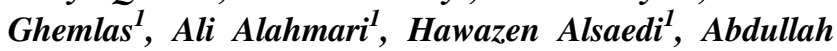
Aljefri $^{1}$, Yiskah Simons ${ }^{1}$, Viqaruddin Mohammed ${ }^{1}$, Syed Jafri', Zebaida Ibrahim ${ }^{1}$, Areej Ali ${ }^{1}$, Neameh Farhan', Sawsan Abu Awwad', Diana Bushnak', Adel Alhmouz', Bouchra Safi, Khaldoun Alshorman ${ }^{1}$

${ }^{1}$ King Faisal Specialist Hospital and Research Center, Riyadh, Saudi Arabia

Background: Hematopoietic Stem Cell Transplantation (HSCT) is often offered as curative and / or supportive therapeutic option to patients with cancers, blood disorders and other conditions that requires coordinated pre- and posttransplant care involving series of investigations and / or evaluations. Pediatric Stem Cell Transplantation program at King Faisal Specialist Hospital \& Research Centre, Riyadh as part of the Pediatric Hematology / Oncology department is the national referral center for children ( $\leq 14$ years of age) indicative of HSCT, performing an average of 130 transplants per year making it the largest and most experienced pediatric transplant program within the country providing services through one of its consolidated sub-specialized programs: Oncology,bone marrow failures, hemoglobinopathies, immune disorders and metabolic disorders; program is accredited by the Joint Accreditation Committee ISCR- Europe \& EBMT(JACIE).

Methods: Weekly HSCT coordination meetings are conducted every Sunday afternoon to discuss post- HSCT care of patients on floor,in addition to pre-transplant list, new referrals work-up and / or clearance of prospective transplant patients and admission plan of cleared patients divided by alternating sub-specialty on a weekly basis i.e. oncology, non-malignant blood disorders, Immune \& metabolic disorders exclusive of urgent transplant cases.
Results: Presence from attending physicians, clinical nurse coordinators, charge nurses, quality coordinator and front-line staff resulted in effective Pre-HSCT admission planning with priortisation of patients to better utilize inpatient resources in preparation for HSCT such as Hickman line and TBI slots, further early pre-HSCT work-up planning was observed in malignant and non-malignant blood disorder cases on floor. Patients from waiting- lists were weekly reviewed by the team to facilitate investigations and / periodic alternate donor search was conducted for patients with no-matched donors and an increase by $90 \%$ (2018 vs.2017) in transplants of patients with alternate donors was observed when first three quarters of 2018 compared to that of 2017, while an increase in total number of transplants by 14\% was observed (2018: 112 transplants vs. 2017:98 transplants).Post-HSCT care discussions resulted in early identification of complications during transplantation course(conditioning phase) on floor to avoid extended hospitalization; effective discharge planning including but not limited to procedural \& clinics scheduling and consultations was observed.

Conclusions: In our practice weekly interdisciplinary round / meeting with team members involved in providing pre- and post-HSCT sub-specialized care enabled a system of effective communication and prioritization for urgent transplants and patient-centered social issues were also addressed . Furthermore, it was realised the sub-specialised format of meetings enabled focused discussions resulting in prompt decisions otherwise delayed by scheduling limitations however, additional analysis to better understand the effect of this coordination meeting in terms of hospital metrics and patient satisfaction is warranted.

Clinical Trial Registry: N/A

Disclosure: None to disclose

\section{NP054}

Effect of central venous access device workshop on knowledge of nurses

\section{Janhavi Alim ${ }^{1}$, Meera Achrekar ${ }^{1}$}

${ }^{1}$ Tata Memorial Centre Advanced Centre for Treatment, Research and Education in Cancer Navi Mumbai, India

Background: Central venous access device (CVAD) are indispensable while caring for a patient requiring for long treatment specially in a cancer care center. Nurses practicing in oncology setting should have knowledge and skill regarding the care and management of CVAD, which will enable them to understand issues of CVAD and identify/solve problems related to it. Efficient care of CVAD will help to reduce infection, prevent extravasation and phlebitis. A two 
day workshop was organized to update nurse's knowledge and skill. Therefore the objective of this study was to assess the change in knowledge of nurses post workshop.

Methods: A two day workshop with hands on training for central venous access device: care and maintenance was conducted on September 20-21, 2018 at a tertiary care cancer hospital. Pretest-posttest design was used. Fifty-two nurses were selected using Non probability convenient sampling technique. Content validity and reliability was established. Participants were asked to respond to an open ended questionnaire (20 questions) before and after the workshop. Data was analyzed using Descriptive and inferential statistics.

Results: Median age of nurses was 28 years and majority (84\%) were females. Around 61\% had diploma in nursing and $19 \%$ had degree in nursing. Most of the nurses had more than 1 year of experience (median 6 years). None of the participant responded correctly to question on placement and frequency of flushing of Hickman catheter during pretest, which only improved slightly (10\%) in posttest. Only $2 \%$ were able to respond correctly to the question on concentration of heparin required for Hep lock injection which improved to $31 \%$ in posttest. The pretest mean score was 5.2 (S.D=2.1). Post intervention, the mean scores improved to $11.6(\mathrm{~S} . \mathrm{D}=3)$. There was a significant mean difference of 6.5 (95\% CI- 5.7-7.2). There was a positive co-relation with total score and years of experience which was statistically significant $(\mathrm{p}=0.00)$. Mean of pre total score of participants with diploma in nursing was significant compared to the graduates $(\mathrm{p}=0.045)$. It can be concluded that the workshop bought about a statistically significant improvement in knowledge scores of the nurses.

Conclusions: The CVAD workshop helped to improve the knowledge score of nurses. Educational opportunities should be provided as part of in service education to update knowledge and skill of nurses.

Clinical Trial Registry: Not Applicable

Disclosure: Nothing to Declare

\section{NP055}

Nurse's role in the multidisciplinary team for CGVHD and long term complication after HSCT - a case report of a patient with MM and MS

\section{Josipa Belev', Antonela Samardzic ${ }^{2}$, Elizabeta Rendulic ${ }^{1}$, Vesna Druzinic ${ }^{1}$}

${ }^{1}$ University Hospital Centre Zagreb, Zagreb, Croatia, ${ }^{2}$ University of Zagreb, Zagreb, Croatia

Background: Patients after HSCT require long-term care of multidisciplinary team with important role of nurses' care.
At the UHC Zagreb, Croatia, the Multidisciplinary team for cGVHD and long-term complication after HSCT was founded in 2013.

Methods: In this case report we described nursing contribution to comprehensive care of complex patient with multiple myeloma (MM) and multiple sclerosis (MS) after HSCT.

Results: A 55-year-old female was diagnosed with MM and MS in 2013 at the UHC Zagreb. After chemotherapeutic treatment for MM, patient underwent tandem autologous HSCT. In 2016 she progressed into plasma cell leukemia, and received another line of chemotherapy with subsequent allogeneic HSCT which was followed by development of skin GvHD (first acute, then chronic). In 2018, second allo-HSCT was made due to graft rejection, and posttransplantation period was complicated by development of severe acute GvHD (skin and GI) requiring immunosuppression and ECP. During that period patient was pancytopenic, with impaired mobility and with infective complications. The role of nurse in treatment of such complex patient is undeniable because of extensive nursing care required by prolonged hospitalizations. Neutropenia related infections demanded significant engagement and sterile conditions, especially because patient was colonized with multidrug-resistant bacteria. Anemia and thrombocytopenia, together with infection, required long lasting appropriate vascular access for therapeutic intentions, which highlights the need of proper nursing education. Amelioration of nutritive status was required for preservation of muscles. Intensive physiotherapy with muscle strengthening exercises to improve mobility was mandatory. Also, appropriate psychological support was crucial for upgrading quality of life. The patient is currently in remission regarding main disease, with recovered mobility and without need for hospitalization.

Conclusions: The role of nurse in the multidisciplinary team for cGVHD and long-term complication after HSCT treatment is multidimensional, especially in such complex patient as described in this case report.

Disclosure: None

\section{NP056}

Abstract withdrawn.

NP057

Human papillomavirus; the challenge of post-transplant virus, a case study

Angela Leather ${ }^{1}$, Jacqui Stringer ${ }^{1}$

${ }^{1}$ The Christie Hospital, Manchester, United Kingdom 
Background: This case study highlights the challenges of multiple viruses post allogeneic transplant. This 49 year old, highly educated, lady was diagnosed with $\mathrm{Ph}+$ ve $\mathrm{B}-\mathrm{ALL}$ in 2015, transplant was delayed due to neutropenic sepsis, delayed BM recovery and poor performance status. Human Herpes Virus 6 (HHV6) detected at low level pre BMT with unknown clinical significance. An unrelated (male) 10/10 matched but CMV (discrepant) Pos/Neg donor was used for BMT in March 2016 with FMC conditioning, 2 weeks post BMT, patient reactivated CMV and during 2.5 years experienced multiple reactivations treated with wide range of antivirals, complicated by chronic renal damage, yet continues with an ongoing morphological remission with chronic GVHD of mouth, eyes and skin.

Methods: Referral to Women Health Clinic was requested in March 2018, as suspected vvGvHD, Patient complained of itching and dryness, vulva majora had white plague like lesions, swabs returned with Streptoccus B infection. Standard antibiotics not tolerated and topical treatments unsuccessful. Referred to gynaecology due to obvious progression. Vulval biopsy confirmed HPV type 59 and Vulval intraepithelial neoplasia (VIN2), cervical biopsy however was negative. Following MDT review, radical vulvectomy and laser treatment was recommended. Patient explored available literature for less extreme management, contacting several clinicians worldwide for trial options including off-licence topical preparation. This was used for 3 weeks but the affected area had increased significantly in this time, up to and including the anal cleft. In-house clinical collaboration and an in-depth literature review facilitated development of a bespoke essential oil cream to target the HPV 59 prior to possible surgery for VIN2. Cream commenced 22nd October 2018, to a set protocol.

Results: Within the first week of application, the cream had minimised symptoms of itching and discomfort associated with the vesicles and there had been no further spread. Slow dose escalation was commenced. There will be careful monitoring and reporting of progress in the final presentation.

Conclusions: Transplant patients often have complex / poor immune functioning making management of posttransplant viral complications challenging, necessitating extreme measures such as radical vulvectomy due to the consequent VIN2. This surgery would not ultimately address the underlying viral load, therefore the clinical conundrum remains. This situation had been highlighted previously in the hospital (2014), when a male patient was diagnosed with multiple resistant genital warts, histology Condyloma Acuminatum (penile HPV). He underwent several excisions and lazar ablations between May \& November 2014, at which point the surgeons were reporting extensive progression (mostly of the glans and anal cleft) and he underwent a glansectomy with split-skin graft. In February 2015, the surgeons were still reporting the warts were unchanged and several more surgical procedures were required to manage the situation.

This case study highlights the impact of one of the challenges faced by clinical teams working with patients post allograft; their compromised ability to flight viral infections. It shows the importance of working collaboratively in the face of complex clinical issues to facilitate best patient outcomes.

Disclosure: Nothing to declare

\section{NP058 \\ Implementation of a survivorship program for allogenic HCT across a network of transplant centers}

Lisa Scholl, Betsy Blunk, Caitlin Reyes ${ }^{1}$, Darren Johnson', Keith Byram ${ }^{3}$, Carole Elledge ${ }^{4}$, Kate Regan', Kathleen Scaggs ${ }^{2}$, Rocky Billups ${ }^{1}$, Tonya Cox ${ }^{2}$, Carlos Bachier $^{I}$, C. Fred Le Maistre ${ }^{I}$, Minoo Battiwalla ${ }^{I}$

${ }^{1}$ Sarah Cannon Blood Cancer Network, Nashville, TN, United States, ${ }^{2}$ Sarah Cannon Blood Cancer Network, Denver, CO, United States, ${ }^{3}$ Sarah Cannon Blood Cancer Network, New Orleans, LA, United States, ${ }^{4}$ Sarah Cannon Blood Cancer Network, San Antonio, TX, United States, ${ }^{5}$ Sarah Cannon Blood Cancer Network, Austin, TX, United States

Background: The unique needs of the rapidly increasing numbers of allogeneic HCT survivors have stimulated the establishment of long-term follow up (LTFU) / survivorship programs. Survivorship programs are not only mandated by quality organizations such as FACT/JACIE but have also been associated with superior clinical outcomes. Such initiatives require leadership prioritization to support dedicated resources, multidisciplinary interaction and specialized staffing. While excellent survivorship programs do exist at the single-institution level, there has been no multicentric programmatic model. We report our implementation of a survivorship program across the Sarah Cannon Blood Cancer Network (SCBCN) of six stem cell transplantation centers with a combined annual volume of $>1,000$ transplants.

Methods: Literature search did not identify publications on models for multicentric survivorship clinics. An internal task force consisting of Advanced Practice Professionals (APPs), physicians, SCBCN administration, pharmacists, and LTFU coordinators met twice monthly over a year to determine program structure. Site capabilities were mapped, care guidelines developed, and expert guidance was integrated. Time studies were used to develop metrics for the utilization of survivorship LTFU nurse coordinators. A business case was developed and recommendations implemented.

Results: We identified several challenges that were attributable to the heterogeneity in interest, existing staffing, capabilities and administrative support at each site. The final 
model was designed to be flexible, scalable, financially viable and capable of delivering quality care.

\begin{tabular}{ll} 
The Sarah Cannon Survivorship Clinic model \\
\hline $\begin{array}{l}\text { Establish survivorship } \\
\text { milestones }\end{array}$ & $\begin{array}{l}\text { Distinguish routine care visits from } \\
\text { those dedicated to survivorship (at } \\
\text { day 100, } 6 \text { months, annually) }\end{array}$ \\
$\begin{array}{l}\text { Consultative } \\
\text { multidisciplinary model }\end{array}$ & $\begin{array}{l}\text { Dinguish routine care visits from } \\
\text { day } 100,6 \text { months, annually) }\end{array}$ \\
Critical staffing roles & $\begin{array}{l}\text { 1. LTFU transplant coordinator } \\
\text { nurse } 2 \text {. APP }\end{array}$ \\
Survivorship visit & $\begin{array}{l}\text { Pre-visit questionnaire-> } \\
\text { comprehensive visit with APP -> } \\
\text { integration with additional } \\
\text { information at monthly survivorship } \\
\text { rounds } 4 \text { standardized template for } \\
\text { final recommendations to patient and } \\
\text { multidisciplinary partners }\end{array}$
\end{tabular}

Future directions

"Integration with Stafa CT Individualized survivorship care (enterprise transplant software)" plan, track key metrics, research

Guidelines

Periodically refresh guidelines

[[NP058 Table] 1. The Sarah Cannon Survivorship Clinic model]

The Sarah Cannon Survivorship Clinic model is being implemented across the network. Critical positions have been established at each center for a dedicated LTFU nurse for coordination of scheduling and communication as well as an APP to independently staff the milestone visit. Regular survivorship rounds follow a structured process to facilitate comprehensive evaluation and communication to the multidisciplinary team. Standardized guidelines have been jointly developed and training is spreading from a pilot site to other centers in the network.

Conclusions: The Sarah Cannon Survivorship Clinic model is a scalable solution that addresses the critical requirements for transplant survivorship across multiple centers.

Clinical Trial Registry: N/A

Disclosure: Nothing to declare

\section{NP059}

Fear of disease progression as key factor for the prediction of psychosocial distress in hematological cancer patients prior to HSCT

\section{Sandra Thiele ${ }^{1}$, Nicolaus Kröger ${ }^{2}$, Anya Pedersen ${ }^{1}$, Simone Goebel ${ }^{1}$}

${ }^{1}$ Christian-Albrechts-Universität zu Kiel, Clinical Psychology and Psychotherapy, Kiel, Germany, ${ }^{2}$ University Medical Center Hamburg-Eppendorf, Hamburg, Germany
Background: Despite the high objective risks associated with hematopoietic stem cell transplantation (HSCT), Fear of Disease Progression ( $\mathrm{FoP}$ ) has only sparsely been studied in patients with leukemia prior to HSCT.

Methods: We included adult leukemia patients prior to their first HSCT ( $n=63,61.9 \%$ male). Patients were tested within a week prior to HSCT during in-patient stay. They completed self-report measures assessing psychosocial distress (NCCN Distress Thermometer) and Fear of Disease Progression (Fear of Progression Questionnaire-Short Form, FoP-Q-SF). Additional measures were included for the assessment of possible predictors and clinical correlates of FoP (e. g., patients' physical state, depression, or social support).

Results: FoP is a relevant predictor of psychosocial distress (medium effect, $p=.001$ ). Compared to other patient groups and stages in the disease trajectory, patients exhibited major levels of FoP. Thus, 23 patients (36.5\%) suffered from high FoP (i. e., scored $\geq 34$ in FoP-Q-SF). According to the 12 items of the FoP-Q-SF, some of the greatest fears reflected common fears of cancer patients (e. g., fear about leaving their families behind). Other content of FoP however reflected specific fears related to the extreme situation prior to HSCT (e. g., fear of severe medical treatments during the course of the illness). The following medical or sociodemographic characteristics were associated with high levels of FoP: previous cancer diagnosis, diagnosis of an acute leukemia, and lower education. Patients with higher levels of FoP were more depressed, more anxious, reported reduced physical functioning and lower positive social support.

Conclusions: This was the first study providing an indepth analysis of prevalence, content and clinical correlates of FoP in leukemia patients prior to the extreme situation of HSCT. FoP is frequent and of major clinical importance in hematological cancer patients prior to HSCT. As FoP cannot be predicted by objective patients' characteristics, the routine screening for FoP is recommended.

Disclosure: Nothing to declare.

\section{NP060}

Re-engineering follow-up care after allogeneic stem cell transplantation: Prototype development for an ehealthsupported model of care- the smile study

\section{Lynn Leppla ${ }^{1,2}$, Sabine Valenta ${ }^{1,3}$, Alexandra Teynor ${ }^{4}$, Sabina De Geest ${ }^{1,5}$}

${ }^{1}$ University of Basel, Basel, Switzerland, ${ }^{2}$ Freiburg University Medical Center, Freiburg, Germany, ${ }^{3}$ University Hospital Basel, Basel, Switzerland, ${ }^{4}$ University of Applied Sciences Augsburg, Augsburg, Germany, ${ }^{5}$ Academic Centre for Nursing and Midwifery, Leuven, Belgium 
Background: Re-engineering Follow-up Care after Allogeneic Stem Cell Transplantation (HSCT) is needed due to an increasing number of long-term survivors in need of comprehensive care needs. eHealth solutions are promising in improving clinical outcomes in cancer patients. Yet successful implementation and sustainability of innovative eHealth supported care models requires application of implementation science methods (e.g. contextual analysis, technology openness assessments) as well as a user centered design for technology development. We report on the development, of the first prototype of an Integrated model of care in hematopoietic $\underline{\text { SteM }}$ cell transplantatIon faciLitated by eHealth (SMILe)

The overall objective is the context and theory-driven development of a first prototype (SMILe-V1) of an integrated model of care in in the HSCT setting facilitated by eHealth technology.

Methods: A single-center explanatory sequential mixed methods design was used in one German HSCT to map out the context and understand patients and clinicians perspective on the current follow-up situation and possible eHealth support. 60 patients and 5 clinicians were surveyed and 10 respectively 11 interviewed. Based on the findings and external evidence by using Michie's Behavior-ChangeWheel, intervention content for the SMILe-V1 prototype was developed. An agile software development approach with user centered design was applied to transfer the content into a user-friendly technology.

Results: SMILe-V1 consists of two elements: a) a carecoordinator, and b) the SMILe-platform which includes an App for patients and a web interface for the care-coordinator. The care-coordinator delivers face-to-face intervention modules which will subsequently facilitated by technology. Topics emerged out of the context analysis and resulted in the following modules: electronic monitoring, medication adherence, infection prevention and physical activity. By using the Behavior-Change-Wheel we mapped patients and clinician's perspective as well as the existing literature upon each topic to define the target behaviors, define what needs to change, choose effective behavior change techniques and decide the mode of delivery for SMILe-V1.

The SMILe-platform connects each patient with the carecoordinator in the transplant center and allows electronic monitoring by collecting the most important medical, behavioral and psychosocial parameters. Parameters have been defined by a group of experts across the Austria, Switzerland and Germany. Patients receive automated feedback based on predefined algorithms defined by the attending physicians of our outpatient clinic. Further, patients receive assistance in recognizing symptoms or early signs of complications. By exceeding or undercutting, the defined cut-offs the app produces orange, red and dark red alarms to both patients and care-coordinators in the center and gives advice how to act upon the entered data using predefined algorithms and protocols for the carecoordinator. Beside, patients can visualize their parameter trajectories and also receive short self-management instructions. User-test throughout the development phase resulted in constant high system usability scores of $>75$.

Conclusions: The use of implementation science methods combined with a user centered design as well as theory driven intervention development provide a solid basis of the SMILe-V1 that will be tested in a first randomized clinical trial in 2019.

Disclosure: Nothing to declare

\section{NP061 \\ The use of lollipop in the nausea and vomiting prevention during the infusion of cryopreserved peripheral blood stem cell}

Silvia Sangüesa Domínguez, Maria Cristina González Rodríguez $^{I}$, Cristina Martín Benito ${ }^{I}$, Francisco José Rodríguez Alcazar', Rosa María Cortés Pinilla', Olga González García ${ }^{l}$

${ }^{1}$ Complejo Asistencial Universitario de Salamanca, Salamanca, Spain, ${ }^{2}$ Gerencia de Atención Primaria de Salamanca, Salamanca, Spain

Background: Nausea and vomiting are the most frequently and distressing side effects for the patient during the infusion of autologous peripheral blood stem cell transplantation (PBSC).

Dimethylsulfoxide (DMSO) is used as a cryoprotectant in the preservation of PBSCs, when infused with the thawed cell suspension, has a great capacity to cross the tissue membranes and within a few minutes of administration, patients begin tasting like cockles-like odor. The taste and odor are associated with the metabolites of DMSO which affect the central limbichypothalamic pathways that cause nausea and vomiting.

The principal objective is to evidence the relation between the administration of a lollipop and the reduction of nausea and vomiting during the infusion of PBSC.

Methods: A prospective, randomized and controlled study was conducted to evaluate the presence of nausea and vomiting during the PBSC infusion process carried out from September 2017 to October 2018. Seventy patients were included in the study, divided into two groups, the group 0 received a lollipop and the group 1 did not. Patients with repeated nausea or vomiting before infusion who received additional antiemetics were excluded from the study. Age, sex, diagnosis, conditioning regimens, total DSMO received, total symptoms, total nucleated cells, CD34 number, total volume infused and infusion rate were analyzed with 
descriptive and multivariate analysis. All patients were informed and gave their prior consent.

Results: The group 0 with thirty seven patients $\left(n_{0}: 37\right.$, average age: 53.24, range: $25-70$ ) who received a lollipop during infusions and group 1 with thirty three patients $\left(\mathrm{n}_{1}\right.$ : 33, average age : 54.90, range: $25-71$ ) did not. The administration of midazolam as pre-medication presents a significant difference between the groups $\left(37.8 \% \mathrm{n}_{0}=14\right.$ versus $\left.60.6 \% \mathrm{n}_{1}=20, \mathrm{p}=0.057\right)$ and a relationship of dependence between midazolam and nausea ( $p$-value $=$ 0.004 ), it was decided to remove these patients from the study. Therefore, group 0 had less nausea with a significant difference $\left(17.4 \% \mathrm{n}_{0}=4\right.$ versus $46.2 \% \mathrm{n}_{1}=6$, $\mathrm{p}$-value $=$ 0.064). In the multiple correspondence analysis performed on the groups, the presence of nausea and vomiting, the intervals of total DSMO received and the rhythm of infusion presents two dimensions. (Graph 1). The logistic regression model performed has a good goodness of fit (Hosmer and Lemeshow test $\mathrm{p}=0.829$ ), explains $33.7 \%$ $\left(\mathrm{R}^{2}\right.$ Nagelkerke) of the nausea variance and correctly classifies $77.8 \%$ of the cases. The group (OR 11.502 (95\% CI $1.204-109.867) ; \mathrm{p}=0.034)$ and $\operatorname{sex}(\mathrm{OR} 0.080(95 \% \mathrm{CI}$ $0.008-0.811) ; \mathrm{p}=0.033$ ) were the only variables that were associated independently with the absence of nausea.

Conclusions: Male patients who did not receive midazolam and no lollipop were more likely to have nausea, decreasing in that group this probability to almost 50\% when taking the lollipop, so we conclude that the use of a lollipop during the infusion of autologous Cryopreserved PBSCs may be promising to reduce nausea and vomiting with a low cost and easy administration.In any case, we will continue with the study to increase the sample of patients and confirm the results obtained so far.

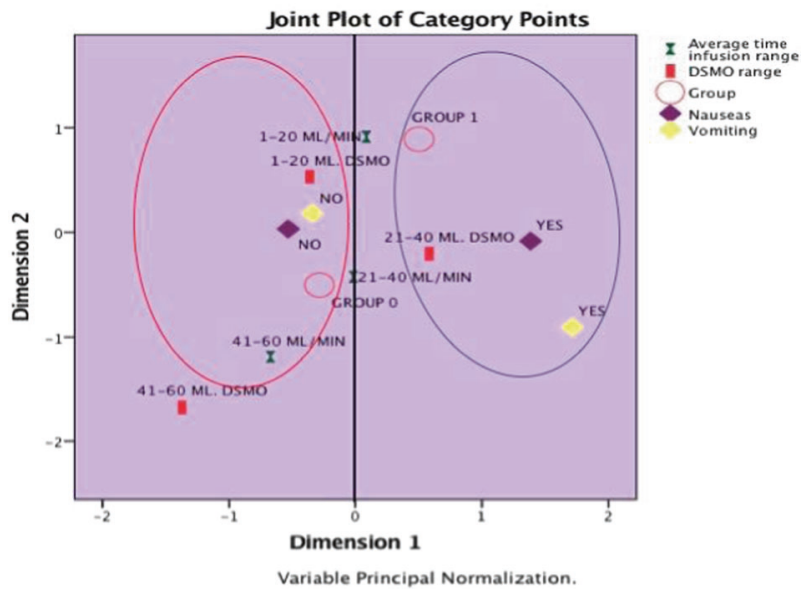

[[NP061 Image] 1. Graph 1. Multiple correspondence analysis.]

Disclosure: Nothing to declare

\section{NP062}

Nutritional management in mitochondrial neurogastrointestinal encephalopathy (MNGIE) undergoing haematopoietic stem cell transplant (HSCT)

\author{
Nicola Turner-Smith ${ }^{1}$, Robert Wynn ${ }^{2}$ \\ ${ }^{1}$ Royal Manchester Children's Hospital, Manchester, Uni- \\ ted Kingdom, ${ }^{2}$ Royal Manchester Children's Hospital, \\ Manchester, United Kingdom
}

Background: Mitochondrial Neurogastrointestinal Encephalopathy (MNGIE) is an autosomal recessive multisystem disease caused by mutations in the gene encoding thymidine phosphorylase (TP). The resulting TP deficiency leads to an accumulation of thymidine and deoxyuridine, causing mitochondrial dysfunction. Limited research suggests that HSCT may be an effective treatment by restoring biochemical abnormalities. Peripheral neuropathy, opthalmoplegia, leukoencephalopathy and gastrointestinal dysfunction, including dysmotility, pain, cachexia and postprandial emesis, are characteristic, with sub-optimal nutritional status. Intensive nutritional support, often Parenteral nutrition (PN), is needed to effectively support outcomes. This case considers the nutritional management of a 13 year old girl with MNGIE undergoing HSCT. She is the 3rd of 5 siblings (4 have the diagnosis of MNGIE) and had early disease presentation, having been screened after later presentation in the older siblings. All 4 have now undergone HSCT with the healthy sibling being the donor.

Methods: In July 2018, this girl was admitted for HSCT. Her nutritional status was very poor (weight $<0.4$ th centile, height 2nd-9th centile, BMI significantly $<0.4$ th centile). Appetite was poor, and she experienced frequent episodes of nausea and abdominal pain. Prior to admission oral nutritional supplements $(1.5 \mathrm{kcal} / \mathrm{ml}$ and $2.4 \mathrm{kcal} / \mathrm{ml}$ whole protein supplements) offered some efficacy in achieving weight gain - tracking $<0.4$ th centile. Nasogastric feeding (NGF) was initiated during the conditioning phase of transplant, using a $1 \mathrm{kcal} / \mathrm{ml}$ peptide feed, feeding to approximately 50\% Estimated Average Requirements (EAR). This was tolerated for 4 days but had to be stopped and PN started on day 1 post-transplant due to increased vomiting, abdominal pain and diarrhoea. PN was required until 27 days post-transplant when we began transitioning from PN to NGF. Peptide feed proved intolerable due to vomiting, abdominal pain and loose stools. Amino acid feed, concentrated to $1.26 \mathrm{kcal} / \mathrm{ml}$, was tolerated with pharmacological support using anti-emetics and prokinetics. Volumes were gradually increased to $75 \%$ EAR and achieved stable weight.

Results: On discharge, weight remained tracking $<0.4$ th centile and stable off PN. NGF continued with an 
improvement in gastrointestinal symptoms over the following 2 months. To date, appetite remains minimal but small quantities of oral diet are tolerated, allowing a reduction in feed to $65 \%$ EAR. Reports indicate that PN is frequently required in MNGIE due to the very poor tolerance of enteral nutrition (EN). The elder 2 siblings in this case both require continuing home $\mathrm{PN}$ following transplant. They presented with more severe symptoms and poorer nutritional status. Disease onset is insidious and improvement in gut pathology after BMT is also slow. This report shows the potential for effective nutritional management with amino acid feed. This would offer significant benefits in reducing risks associated with long term PN.

Conclusions: There is little reported on the nutritional management of MNGIE, specifically post HSCT. This case is significant, suggesting that EN can be tolerated in the early post-transplant stage in the younger Paediatric patient - perhaps with less severe disease presentation. Longer term nutritional outcomes are not yet known - further research into enteral feed types and mode of feed delivery would be beneficial.

Disclosure: Nothing to Declare

\section{NP063}

Oral mucositis in autologous stem cell transplantation for solid tumors in children: Comparison between single and tandem procedures

Oana Otilia Niculita ${ }^{1,2}$, Cristina Georgiana Jercan ${ }^{1,2}$, Anca Colita ${ }^{1,2}$

${ }^{1}$ Fundeni Clinical Institute, Bucharest, Romania, ${ }^{2}$ Carol Davila University of Medicine and Pharmacy, Bucharest, Romania

\section{Background:}

Introduction: Solid tumors represent $30 \%$ of all pediatric cancers, excepting brain tumors. The management of solid tumours is complex and includes chemotherapy, surgery, radiotherapy and in high-risk cases stem cell transplantation (SCT).Oral mucositis(OM) is the most frequent complication of pediatric patients undergoing SCT. In transplant recipients OM can have long-term evolution, sometimes severe.

Aim: We analyzed the results of pediatric patients with solid tumours and HSCT treated in Fundeni Clinical Institute between January 2002 and October 2018 to evaluate the median duration of OM episode and grade of severity according to the numbers of procedures performed.

Methods: We retrospectively analyzed patients with solid tumours and HSCT. Patients diagnosis were: neuroblastoma (NBL), germ-cell tumour (GCT), Ewing sarcoma, nefroblastoma. Patients were divided into 2 groups: first group with one HSCT and second groupwith more than one HSCT. Criteria used to asess severity of OM were, according to WHO: Grade 1 - soreness \pm erythema, Grade 2 - erythema, ulcers; patient can swallow solid food, Grade 3 ulcers with extensive erythema; patient cannot swallow food, Grade 4 - mucositis to the extent that alimentation is not possible. All patients performed prophylactic measurements to prevent OM : mouth cleansing with sodium bicarbonate solution, mouthwash with clorhexidine, oral spray with Zinc-Taurine complex and polyvinylpyrrolidon. All patients received antibiotic, antiviral and antifungal prophylaxis, parenteral nutrition and supportive treatment. All parents signed informed consent forms.

Results: In our clinic, between January 2002 and October 2018, were performed 67 auto-HSCT procedures to 52 patients: $34(65.3 \%)$ pts with NBL, $9(17.3 \%)$ pts with ES, 6 (11.6\%) pts with GCT and $3(5.8 \%)$ pts with nefroblastoma. Patients were divided into 2 groups: first group with a single SCT procedure (79\% of pts) and second group with more than one SCT procedure (21\% of pts). Patients were diagnosed, staged and received treatment according to international protocols. Sex ratio was 18F/34M. Age distribution was $1-4$ y $38 \%$ (20 pts), $4-10$ y $35 \%$ (18 pts), > 10 y $27 \%$ (14 pts). Distribution of patients according to grade of OM is represented in Figure 1.

The median duration of OM in the first group was 6 days (range 3 to 17 days), while in the second group was 13 days (range 3 to 21 days). For the first group patients received pain medication for an average of 7 days, whereas for the second group the period increased to 13 days. Also, for the second group we noticed the engraftment period increased, along with the hospitalization and supportive measures. Patients with multiple courses of chemotherapy and with multiple hospitalizations presented increased infectious risk and during the second procedures developed various infectious complications. Patients from both groups presented full recovery of oral mucositis by the time they were discharged.

Conclusions: There are no correlations between the severity of OM and age or sex of patients. Duration of OM episode is increased in patients with multiple SCT. The incidence of severe OM increased from $17 \%$, after the first transplant to $69 \%$ after the second transplant.

Disclosure: Nothing to disclose

\section{NP064 \\ Radiation recall: special nursing care in patients previously irradiated for the neuroendocrine cancer underwent high dose chemotherapy and autologous HSCT}

Yu Fang Chu', Jia Hau Liu ${ }^{1}$, Jui Yi Chen ${ }^{2}$ 
${ }^{1}$ National Taiwan University, Tai-Cheng Stem Cell Therapy Center, Taipei, Taiwan, Republic of China, ${ }^{2}$ National Taiwan University Hospital, Taipei, Taiwan, Republic of China

Background: Radiation recall is a special clinical phenomenon that local exaggerated tissue necrosis was observed after chemotherapy to the body area where previous radiation were taken place. We herein report our nursing experiences of three patients with such radiation recall immediately after high dose chemotherapy and autologous stem cell transplant. They all had neuroendocrine like tumors, and had previously received local radiation therapy (total dose 2400-3000 cGy) in either the head, neck, abdomen and spine 2 to 7 months before receiving conditioning therapy. After receiving high-dose chemotherapy, the patient developed severe local necrosis to prior irradiated area. The symptoms including: inflammation and ulcer of the skin at the irradiation exposed site, including skin of the back or the skull, grade 3 to 4 oral mucositis but sparing the gastrointestinal tract in head-neck irradiated patients, severe gastrointestinal pain and diarrhea 9 to 15 times/day in D0 to D+2 in previous irradiation area involving the abdomen. The symptoms were all severe which increased nursing care difficulty.

Methods: Nursing for radiation recall complications are recommended in the following 7 approaches: 1 . Collection of treatment history: which aid the prediction of the radiation recall and aid nursing education for the patient and the family before the entering the BMT room for psychological and supportive preparation. 2. Close monitoring: cautious regular physical assessment and symptom management, given that tissue damage may cause increased risk of infection. 3. Skin care: Avoid rubbing and adherence dressing in erythematous skin. Sterile gauze and elastic bandage expanded holding and prevent further skin injury. And water-soluble antibacterial dressing was recommended. This part was the most nursing labor intensive. 4. Oral care: cryotherapy, analgesic mouth rinse, and using appropriate oral cleansing products and antibacterial ointments to relieve the local suffering and prevent secondary infection from oral necrosis. 5. Gastrointestinal symptom care: antidiarrheal agent, use intravenous form of drugs to prevent oral intake of pills. Temporary fasting and total intravenous nutrition support would be also recommended. 6. Pain control: Opioid analgesic such as morphine continuous infusion can effectively relieve pain and diarrhea. 7. Emotional stability and sedation: Severe symptoms can make patients suffering, anxiety, depression or loss confidence for the treatment team. Nurses listening and appeasing as well as using short-acting sedative and hypnotic drugs are necessary.
Results: The three autologous patients successfully pass the severe radiation recall period and gradually relieved their symptoms after 7-10 days of treatment. Proactive symptoms management helped the patients passing over the difficult treatment process.

Conclusions: Radiation recall is a complication that could not be neglected when patient has a previously irradiation area attempts to receive high dose chemotherapy and autologous HSCT. Proper and intensive nursing care is the key for a successful autologous HSCT.

Clinical Trial Registry: Not applicable.

Disclosure: Nothing to declare

\section{NP065}

An unusual case of delayed citrate reaction after peripheral blood stem cell harvest

Haili Cui', Namal Bandara ${ }^{2}$, Michelle Kenyon ${ }^{3}$, Tuula Rintala $^{3}$, Orla Stewart, Donal McLornan, ${ }^{3}$, Victoria Potter $^{3}$, Aleksandar Mijovic $^{4}$

${ }^{1}$ Kings College Hospital NHS Trust, London, United Kingdom, ${ }^{2}$ National Blood Transfusion Service Sri Lanka, Colombo, Sri Lanka, ${ }^{3}$ Kings College Hospital NHS Trust, London, United Kingdom, ${ }^{4}$ King's College Hospital, London, United Kingdom

Background: Autologous Haematopoietic Stem Cell Transplantation (HSCT) is an established treatment for haematological malignancies. Peripheral blood stem cells harvested by apheresis technology from patients in disease remission are widely used in this setting. Standard Anticoagulant Citrate Dextrose solution Anticoagulation (ACDA) is used in this process to prevent blood clotting in the extracorporeal circulation during cell separation. As citrate is an active component in ACDA, mild side effects like peri-oral tingling and numbness are common during the procedure. However, most of the symptoms are transient due to rapid metabolism of citrate in healthy individuals. We describe a case where the symptoms of citrate toxicity developed 30 minutes post-procedure.

Methods: A 69 years old male with Follicular Lymphoma Stage IV diagnosed in 2013, attended the aphaeresis unit for Peripheral Blood Stem Cell Harvest (PBSCH) after a 4 days course of Granulocyte Colony Stimulating Factor (GCSF) injections as per the mobilization protocol. He returned for day 2 collection following $5^{\text {th }}$ dose of GCSF. Spectra Optia machine used for the Hematopoietic progenitor cell collection was set up as standard MNC (mononuclear cells) procedure. The collection was performed using ACDA., total blood volume processed (TBV) was 
$2.5 \mathrm{~L}$ and $1237 \mathrm{ml}$ of ACDA $(0.8 \mathrm{ml} / \mathrm{min})$ was used during the process. Calcium gluconate infusion $(4.5 \mathrm{mmol} / 1$ in $0.9 \%$ Normal saline $30 \mathrm{ml}$ ) at a rate of $20 \mathrm{ml} /$ hour was set up for tingling lips/tongue as per the Standard Operational Procedure (SOP).

Upon completion of collection, standard post-procedure samples; FBC and electrolytes, were taken. Vital signs were stable throughout and patient stayed in the unit for one hour observation as per SOP.

At 30 minutes post-procedure; patient became anxious and reported palpitations. He became pale with cold peripheries and carpopedal spasms were noted. Pulse and blood pressure were unrecordable, but consciousness was maintained. Patient was put into Trendelenburg position, provided Oxygen rebreather and given IV normal saline, Calcium gluconate and IV Magnesium.

Results: Pre-procedure medical assessment revealed no cardiovascular risk factors with normal ECG . Electrolyte levels were normal at pre-assessment and he was alert and well oriented throughout procedure. Fluctuations in his serum electrolytes are given (table 01).

Fluctuations of serum electrolyte levels.

Potassium and calcium levels were maintained within normal range with adequate replacements. However, the patient's low magnesium levels on day 1 further reduced prior to second day collection. This potential cause was identified when the patient became unwell and replacement was commenced.

Conclusions: Symptoms of hypocalcaemia are well recognised by Apheresis nurses. This particular case demonstrates effect of ACDA on Magnesium levels and delayed presentation of symptoms. In the context of elderly patients with poor metabolic regulation it is very important to monitor and replace electrolytes to optimize patient safety during prolonged or repeated cell separation procedures. This is only one case was reported in our Collection Centre.

\begin{tabular}{llllll}
\hline $\begin{array}{l}\text { Electrolytes } \\
(\mathrm{mmol} / \mathrm{L})\end{array}$ & $\begin{array}{l}\text { Pre } \\
\text { assessment }\end{array}$ & $\begin{array}{l}\text { Collection } \\
\text { day 01 Pre } \\
\text { procedure }\end{array}$ & $\begin{array}{l}\text { Collection day } \\
\text { 01 Post } \\
\text { procedure }\end{array}$ & $\begin{array}{l}\text { Collection } \\
\text { day 02 Pre } \\
\text { procedure }\end{array}$ & $\begin{array}{l}\text { Collection day } \\
\text { 02 Post } \\
\text { procedure }\end{array}$ \\
\hline $\mathrm{K}+$ & 4.50 & 3.80 & 3.20 & 3.80 & 3.90 \\
$\mathrm{Ca}+2$ & 2.25 & 2.21 & 2.21 & 2.12 & 1.34 \\
$\mathrm{Mg}+2$ & 0.80 & 0.62 & 0.62 & 0.56 & 0.55 \\
\hline
\end{tabular}

[[NP065 Table] 1. Table 1]

Disclosure: Nothing to declare

\section{NP066}

Incidence rate and risk factors for phlebitis related to the use of peripherally inserted central venous catheter
(PICC) in patients undergoing haematopoietic stem cell transplantation

Nuria Suarez Fernandez ${ }^{I}$, Rubén Sigüenza Vega ${ }^{I}$, Paloma Rojo Jambrina ${ }^{l}$, Rebeca Velasco Fernandez ${ }^{1}$, Rocio Castañon Plasin ${ }^{I}$, Sonia Alonso Fernandez ${ }^{1}$, Maria Jose Quintana Prieto', Ana Julia Gonzalez Huerta', Walter Javier Zambrano Márquez ${ }^{1}$

${ }^{1}$ Hospital Universitario Central de Asturias, Hematology, Oviedo, Spain

Background: Central venous catheters are essential for patients undergoing haematopoietic stem cell transplantation. PICCs are among the different devices available. Despite their many advantages, they are not exempt from complications such as phlebitis

Methods: Retrospective study. All PICC inserted by nursingwere included, from January 2017 to September 2018 in the Hematopoietic Transplant Unit of the Central University Hospital of Asturias. Socio-demographic variables, hospital stay and related to PICC were collected. The phlebitis incidence and incidence rate was calculated. The risk factors were identified using the Odds Ratio

Results: A total of 125 patients were studied, of which 56 patients were inserted PICC, the total number of PICC studied were 63 . The distribution by sex was $65.1 \%$ men and $34,9 \%$ women, with a median age of 58 years (20-70). The distribution according to the type of transplant of patients with PICC was $85.7 \%$ autologous, $4.8 \%$ allogeneic peripheral blood (PB) unrelated, and 9.5\% allogeneic PB relatives. The median duration of the catheters was 18 days (2-61), an incidence of phlebitis 22,2\% and an incidence rate of phlebitis 12.37 per 1000 catheter days. Of the 14 possible diagnostic phlebitis, catheter infection was evidenced in 3 cases, in addition 10 patients presented Bacteremia. Arterial Hypertension (AH) increased the risk of phlebitis (OR $5.5 \mathrm{p} \mathrm{0.01)}$

Conclusions: Arterial hypertension (AH) is positioned as the pathological antecedent of increased risk of developing phlebitis in the PICC, in these cases prevention measures must be intensified. The incidence of phlebitis is higher than that described in the consulted bibliographyIt should be noted that no patient had thrombosis, one of the main complications associated with this type of catheter

Disclosure: Nothing to declare

\section{NP067}

Working group engaged in the drafting of an unique document concerning the parameters of evaluation of child's problematic issues

\section{Alessandra Buccino ${ }^{1}$, Rosanna Ceresoli ${ }^{1}$}


${ }^{1}$ ASST Spedali Civili Brescia, Brescia, Italy

Background: The child who is subjected to conditioning chemoterapy and TCSE(hematopoietic stem cell transplantation) needs of a constant monitoring of the vital parameters and the risk factors at all stages of its treatment path. In our field we have felt the necessity to have a single document where we can write down all the data useful to evaluate constantly the complications and criticalities concerning the child subjected to TCSE in a non-repetitive way.

Methods: For the drafting of the new document we started from reviewing existing protocols and business procedures, deepening knowledge thanks to the published literature on the subject and evaluating single-sheet budgets of other critical and sub-intensive units.

Results: The document must allow the observer to have a unique clinical picture, immediate and complete on vital parameters, revenue balance outputs, VOD-GvHD risk and signs and symptoms such as pain, nausea, vomiting and pruritus. All the professional figures that are part of the care team must have easy access to the document, both for the comprehension and for the compilation.

Conclusions: We managed to get a document that fulfilled all our needs and that, at the same time was easy to fill out, adding also an explanation to make it more complete and usable.

Disclosure: Nothing to declare

\section{NP068}

\section{Pain diary keeping compliance of inpatients' parents}

Julia Oparina ${ }^{I}$, Olga Ivanova ${ }^{I}$, Ekaterina Goncharova ${ }^{I}$, Maxim Bogomolny, Lyudmila Zubarovskaya, Boris Afanasyev $^{I}$
${ }^{1}$ R.M.Gorbacheva Memorial Institute for Pediatric Hematology, St. Petersburg, Russian Federation

Background: Self-filled diaries kept by a patient or caregiver are an effective method for clinical dynamics evaluation in both outpatient and inpatient settings. The realtime data collection is better for pain intensity monitoring than retrospective data analysis for each 24-hour period as the latter is biased by a number of factors such as variability in pain intensity or change in pain's emotional perception when filling the questionnaire from memory. Due to this range of problems associated with the latter, we conducted a study aimed at diary keeping compliance in pediatric inpatients.

Methods: A total of 64 pediatric patients aged 2.4 to 17 years supervised by pain management team were given cards with Wong-Baker face scale and visual analogue scale with additional diaries for baseline and breakthrough pain assessment. These diaries were filled in each 6 hours during the all analgesia period. The median follow-up period was 8 days.

Results: The overall compliance was $72 \%$. All patients were divided into 4 groups according to diary-filling compliance degree. The $0-25 \%$ group consisted of 25 patients with median age of 6 years, the $26-50 \%$ group of 9 patients with median age of 7 years; the 51-75\% group contained 6 children with median age of 10 years, and 76-100\% 33 children with median age of 9 years.

Conclusions: The results obtained may indicate that an influence of patients' age on his/her caregivers pain diary keeping compliance. This may be due to communicative abilities for children of different age. For more precise conclusions an additional research is required.

Disclosure: No 